\title{
RANDOM CURRENT REPRESENTATION FOR TRANSVERSE FIELD ISING MODEL
}

\author{
NICHOLAS CRAWFORD AND DMITRY IOFFE
}

\begin{abstract}
Random current representation (RCR) for transverse field Ising models (TFIM) has been introduced in [15]. This representation is a space-time version of the classical RCR exploited by Aizenman et. al. [1,3,4]. In this paper we formulate and prove corresponding space-time versions of the classical switching lemma and show how they generate various correlation inequalities. In particular we prove exponential decay of truncated two-point functions at positive magnetic fields in z-direction and address the issue of the sharpness of phase transition.
\end{abstract}

\section{The MODEL AND THE RESUlts}

In what follows, we shall, for brevity, consider translation invariant models on $\mathbb{Z}^{d}$. Specifically, let $\mathbb{T}_{N}$ be the $d$-dimensional lattice torus of linear size $N$ and $\mathbf{J}=\left\{J_{i j}=J_{i-j}\right\}$ is a finite range irreducible translation invariant interaction. Let $h \geq 0, \rho>0, \lambda \geq 0$ and $0 \leq \beta \leq \infty$. The quantum Hamiltonian we are going to consider is of the form,

$$
-\mathcal{H}_{N}=\frac{\rho}{2} \sum_{i, j} J_{i j} \hat{\sigma}_{i}^{\mathrm{z}} \hat{\sigma}_{j}^{\mathrm{z}}+h \sum_{i} \hat{\sigma}_{i}^{\mathrm{z}}+\lambda \sum_{i} \hat{\Sigma}_{i}^{x} .
$$

Above $\hat{\Sigma}^{x}=\left(I+\hat{\sigma}^{\times}\right) / 2$, and $\hat{\sigma}^{\mathrm{z}}$ and $\hat{\sigma}^{\times}$are usual Pauli matrices,

$$
\hat{\sigma}^{z}=\left(\begin{array}{cc}
1 & 0 \\
0 & -1
\end{array}\right) \text {. and } \quad \hat{\sigma}^{\times}=\left(\begin{array}{cc}
0 & 1 \\
1 & 0
\end{array}\right)
$$

Let us introduce the partition function

$$
\mathcal{Z}_{\beta, N}(h, \rho, \lambda) \triangleq \mathrm{e}^{-N^{d} \beta(\rho \bar{J}+h+\lambda)} \operatorname{Tr}\left(\mathrm{e}^{-\beta \mathcal{H}_{N}}\right)
$$

where $\bar{J} \triangleq \sum_{j} J_{i j}$ and we remark that this choice of normalization is made so as to seamlessly introduce certain stochastic integral representations below. Mean values of various local observables are denoted as $\langle\cdot\rangle_{\beta, N}$. For instance,

$$
\begin{aligned}
& \left\langle\hat{\sigma}_{i}^{z}\right\rangle_{\beta, N}=\frac{\operatorname{Tr}\left(\hat{\sigma}_{i}^{z} \mathrm{e}^{-\beta \mathcal{H}_{N}}\right)}{\operatorname{Tr}\left(\mathrm{e}^{-\beta \mathcal{H}_{N}}\right)}, \quad\left\langle\hat{\Sigma}_{i}^{x}\right\rangle_{\beta, N}=\frac{\operatorname{Tr}\left(\hat{\Sigma}_{i}^{x} \mathrm{e}^{-\beta \mathcal{H}_{N}}\right)}{\operatorname{Tr}\left(\mathrm{e}^{-\beta \mathcal{H}_{N}}\right)}, \\
& \text { or, for } i \neq j, \quad\left\langle\hat{\sigma}_{j}^{z} \hat{\Sigma}_{i}^{x}\right\rangle_{\beta, N}=\frac{\operatorname{Tr}\left(\hat{\sigma}_{j}^{z} \hat{\Sigma}_{i}^{x} \mathrm{e}^{-\beta \mathcal{H}_{N}}\right)}{\operatorname{Tr}\left(\mathrm{e}^{-\beta \mathcal{H}_{N}}\right)}
\end{aligned}
$$

This research was supported by a grant from G.I.F., the German Israeli Foundation for Scientific Research and Development. 
Most of the results which we shall derive in the sequel hold uniformly in $\beta<\infty$ and/or in $N$. Whenever this is the the case we shall omit the corresponding subindex. Note that in many cases uniformity in $\beta<\infty$ implies extensions of the corresponding properties to the ground state $\beta=\infty$.

Important quantities to be considered here are the z-magnetization:

$$
M_{\beta, N}(h, \rho, \lambda)=\left\langle\hat{\sigma}_{i}^{z}\right\rangle_{\beta, N},
$$

and the truncated two-point functions,

$$
\left\langle\hat{\sigma}_{i}^{z} ; \hat{\sigma}_{j}^{z}\right\rangle_{\beta, N} \triangleq\left\langle\hat{\sigma}_{i}^{z} \hat{\sigma}_{j}^{z}\right\rangle_{\beta, N}-\left\langle\hat{\sigma}_{i}^{z}\right\rangle_{\beta, N}\left\langle\hat{\sigma}_{j}^{z}\right\rangle_{\beta, N}, \quad\left\langle\hat{\sigma}_{i}^{z} ; \hat{\Sigma}_{j}^{x}\right\rangle_{\beta, N} \text { and }\left\langle\hat{\Sigma}_{i}^{x} ; \hat{\Sigma}_{j}^{x}\right\rangle_{\beta, N} .
$$

Our two main results are:

Theorem A. For every $h>0, \lambda \geq 0$ and $\rho \geq 0$ there exists $c_{1}=c_{1}(h, \lambda, \rho)>0$ and $c_{2}=c_{2}(h, \lambda, \rho)<\infty$, such that

$$
\begin{aligned}
& 0 \leq\left\langle\hat{\sigma}_{i}^{z} ; \hat{\sigma}_{j}^{z}\right\rangle \leq c_{2} \mathrm{e}^{-c_{1}|j-i|}, \quad 0 \leq\left\langle\hat{\Sigma}_{i}^{x} ; \hat{\Sigma}_{j}^{x}\right\rangle \leq c_{2} \mathrm{e}^{-c_{1}|j-i|}, \\
& \text { and, for } i \neq j, \quad-c_{2} \mathrm{e}^{-c_{1}|j-i|} \leq\left\langle\hat{\sigma}_{i}^{z} ; \hat{\Sigma}_{j}^{x}\right\rangle \leq 0 .
\end{aligned}
$$

$B y$ our convention the above results are claimed to be uniform in the torus size $N$ and in $\beta<\infty$.

Theorem B. Uniformly in $h>0, \rho>0$ and $\lambda>0$ the following differential inequalities hold:

$$
M(h, \rho, \lambda) \leq h \frac{\partial M}{\partial h}+M^{3}+M^{2} \rho \frac{\partial M}{\partial \rho}-2 \lambda M^{2} \frac{\partial M}{\partial \lambda}
$$

and,

$$
-\frac{\partial M}{\partial \lambda} \leq \frac{M}{1-M^{2}} \frac{\partial M}{\partial h} \text { and } \quad \frac{\partial M}{\partial \rho} \leq \bar{J} M \frac{\partial M}{\partial h}
$$

Again, by convention, the above inequalities are claimed to hold uniformly in $N$ and in $\beta<\infty$.

In view of the fundamental techniques developed in $[2,3]$, differential inequalities (1.3) and (1.4) imply certain sharpness of phase transition as the transverse field $\lambda$ and/or the inverse temperature $\beta$ are varied. In particular, since $\hat{\sigma}^{z}$ and $\hat{\Sigma}^{x}$ do not commute, the uniformity of our estimates in $\beta$ imply that taking $\beta \rightarrow \infty$, these inequalities still hold and can be used to derive a genuine quantum phase transition, albeit the fact that we derive it using a somewhat classical re-interpretation of the model (see Section 5). In principle, since the model in question could be considered as the strong coupling limit of $(d+1)$-dimensional classical Ising models $[5,9]$, Theorem B could be attempted as a limiting conclusion from the result of [3].

The point of this paper, however, is to try to understand something new; that is to develop general and robust stochastic geometric description of quantum systems, hopefully also yielding simpler, or at least alternative, proofs even in the classical case of $\lambda=0$. In particular, the conclusions of both the theorems above will become rather transparent in the stochastic geometric context which we develop here.

The rest of this paper is organized as follows. Section 2 introduces a recasting of the transverse Ising model in a useful probabilistic language. Further, we set 
down various geometric notions for this recasting which form the basis of our proofs of Theorem $\mathrm{A}$ and $\mathrm{B}$. Section 3 applies these notions to the truncated correlation functions appearing in Theorem $\mathrm{A}$. The resulting expressions may be seen as generalizing the results of the classical Switching Lemma employed in [1,3,4]. Section 4 provides a derivation of Theorem B. Section 5 analyzes expressions for truncated correlations to obtain a proof of Theorem A. Finally, at the end of Section 5 we briefly address the implications for a quantum phase transition in the ground state $\beta=\infty$.

A Bibliographical Remark. Shortly after the first draft of this work was posted on the web, there appeared [8]. The authors of [8] draw motivation from a parity calculus via strong coupling limits for classical RCR, and they develop what they call "random-parity representation" for TFIM. The paper [8] contains very similar formulations and proofs of the corresponding switching lemma and of the differential inequalities. The following bibliographical remark is due:

(a) Although it might look ostensibly different, the random-parity representation of [8] can be readily derived (see Remark 1 below) from the RCR which was introduced in [15] and which we use here. [15] is a transcript of lectures given at Prague's Probability school in 2006.

(b) A simple example of the application to TFIM of the classical switching lemma via limiting parity calculus appears in the Appendix of [10]. This computation was described to one of the authors of [8] in the summer of 2007. There is a long way from this computation to the full "quantum" switching lemma for TFIM, and we had no communications with the authors of [8] regarding the matter since then. In fact, up to the last moment we did not know that the authors of [8] were working on random-current type representations for TFIM.

\section{Stochastic Geometry of The MOdeL}

The stochastic geometric approach to quantum models via the Lie-Trotter product expansion in the imaginary time variable (additional dimension) and a subsequent classical re-interpretation was introduced in [12]. An important mile-stone along these lines is the seminal paper [6]. The approach expounded upon in that paper has many degrees of freedom in the sense that one can experiment with numerous decompositions of the Hamiltonian and with the basis in which the Lie-Trotter expansion is performed to achieve different representations.

We shall skip the derivation of the representation of interest in the present context and proceed directly to its probabilistic description. We refer the interested reader to [15] where the quantum random current representation we are using here was introduced and where various other stochastic geometric descriptions of the transverse field Ising model are discussed at length.

To each site $i \in \mathbb{T}_{N}$ one attaches a copy $\mathbb{S}_{\beta}^{i}$ of the circle $\mathbb{S}_{\beta}$ of circumference $\beta$.

In the ground state case $\beta=\infty, \mathbb{S}_{\infty} \triangleq \mathbb{R}$. The resulting $(d+1)$-dimensional state space of the model is $\mathfrak{S}_{N} \cup \mathfrak{g}$, where,

$$
\mathfrak{S}_{N} \triangleq \cup_{i \in \mathbb{T}_{N}} \mathbb{S}_{\beta}^{i}
$$


and $\mathfrak{g}$ is an artificial "ghost site". The parameters $h, \mathbf{J}$ and $\lambda$ enter the picture in the following fashion: Consider graphs $\mathcal{G}_{N}=\left(\mathcal{V}_{N}, \mathcal{E}_{N}\right)$ with the vertex set $\mathcal{V}_{N}=\mathbb{T}_{N} \cup \mathfrak{g}$, and edge set $\mathcal{E}_{N}=\mathcal{E}_{N}^{0} \cup \mathcal{E}_{N}^{\mathfrak{g}}$ which comprise either edges e $=(i, j) \in \mathcal{E}_{N}^{0}$ with $i, j \in \mathbb{T}_{N}$ and $J_{i-j}>0$, or $\mathrm{e}=(i, \mathfrak{g}) \in \mathcal{E}_{N}^{\mathfrak{g}}$ with $i \in \mathbb{T}_{N}$. As above, we omit the sub-index $N$ whenever it has no impact on the corresponding definition or claim. Let us define the following families of independent Poisson point processes on $\mathbb{S}_{\beta}$ :

Processes of flips. With each e $\in \mathcal{E}_{N}$ we associate a Poisson process $\xi_{\mathrm{e}}$ which has intensity $\rho J_{i-j}$ if $\mathrm{e}=(i, j)$ and intensity $h$ if $\mathrm{e}=(i, \mathfrak{g})$.

Processes of marks. With each $i \in \mathbb{T}_{N}$ we associate a Poisson process $\mathfrak{m}_{i}$ of intensity $\lambda$

In the sequel we shall denote the corresponding product measure as $\mathbb{P}(\mathrm{d} \xi, \mathrm{dm})$. In particular, for notational convenience, whenever there is no confusion the dependence on $(\beta, \mathbf{J}, h, \rho, \lambda)$ will be suppressed. To write down the random current representation we still need to introduce the notion of labels:

Labels. Labels $\nu$ are piece-wise constant maps $\nu: \mathfrak{S}_{N} \mapsto\{r, l\}$. Here $r$ and $l$ are just two symbols, which, if one traces the original derivation of [15], are related to the one particle eigenfunctions in the transverse $\mathrm{x}$-basis. Given a realization $(\xi, \mathfrak{m})$ of the Poisson point processes and a finite subset $A \subset \mathfrak{S}$, let us say that a label $\nu$ is compatible (see Figure 1 )- which will be denoted by $\stackrel{A}{\sim}(\xi, \mathfrak{m})-$ if

(1) $\nu_{i}$ has a jump at $\mathrm{u}$ for every $\mathrm{u} \in A$.

(2) All other jumps of $\nu$ happen at arrival times of $\xi$ : For $\mathrm{e}=(i, \mathfrak{g})$, an arrival of $\xi_{\mathrm{e}}$ enforces a flip of $\nu_{i}$, and, similarly, an arrival of $\xi_{i j}$ enforces a simultaneous flip of $\nu_{i}$ and $\nu_{j}$.

(3) For each $i, \nu_{i}(t)=r$ at each arrival time $t$ of $\mathfrak{m}_{i}$

To facilitate the notation we shall $\operatorname{drop} A$ from $\nu \stackrel{A}{\sim}(\xi, \mathfrak{m})$ whenever $A=\emptyset$.

Representation Formulas. The following formulas are established in [15]: For the partition function (and $\beta<\infty$ ),

$$
\mathcal{Z}_{N}=\int \mathbb{P}(\mathrm{d} \xi, \mathrm{d} \mathfrak{m}) \sum_{\nu \sim(\xi, \mathfrak{m})} \mathbf{1} .
$$

Remark 1. Integrating out the process of marks $\mathfrak{m}$ and calling $r$ "even" and $l$ "odd", one recovers the "random parity" representation of [8].

Given $\mathbf{u}=(i, t)$ define

$$
\hat{\sigma}_{\mathrm{u}}^{\mathrm{z}}=\mathrm{e}^{-t \mathcal{H}} \hat{\sigma}_{i}^{\mathrm{z}} \mathrm{e}^{t \mathcal{H}} \text { and, accordingly, } \hat{\Sigma}_{\mathrm{u}}^{x}=\mathrm{e}^{-t \mathcal{H}} \hat{\sigma}_{i}^{\times} \mathrm{e}^{t \mathcal{H}} .
$$

Note here that the signs match the imaginary time rotation of the quantum evolution. For one- and two-point functions in the $z$ component of spin:

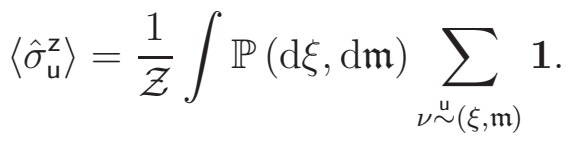


(a)

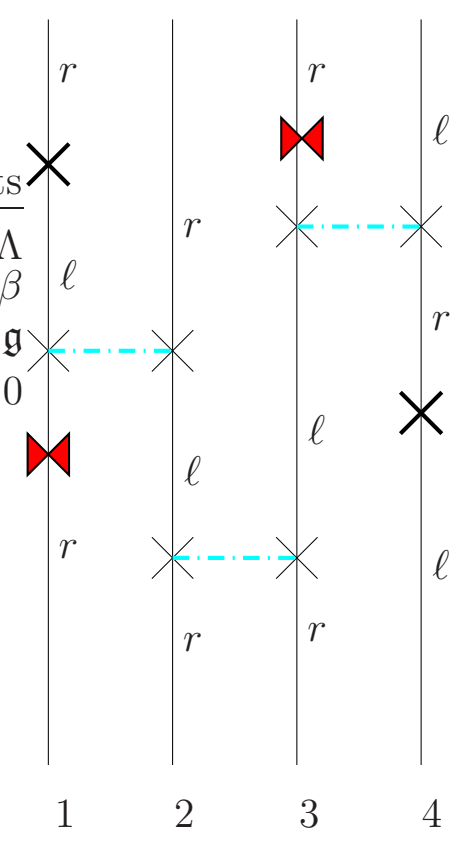

(b)

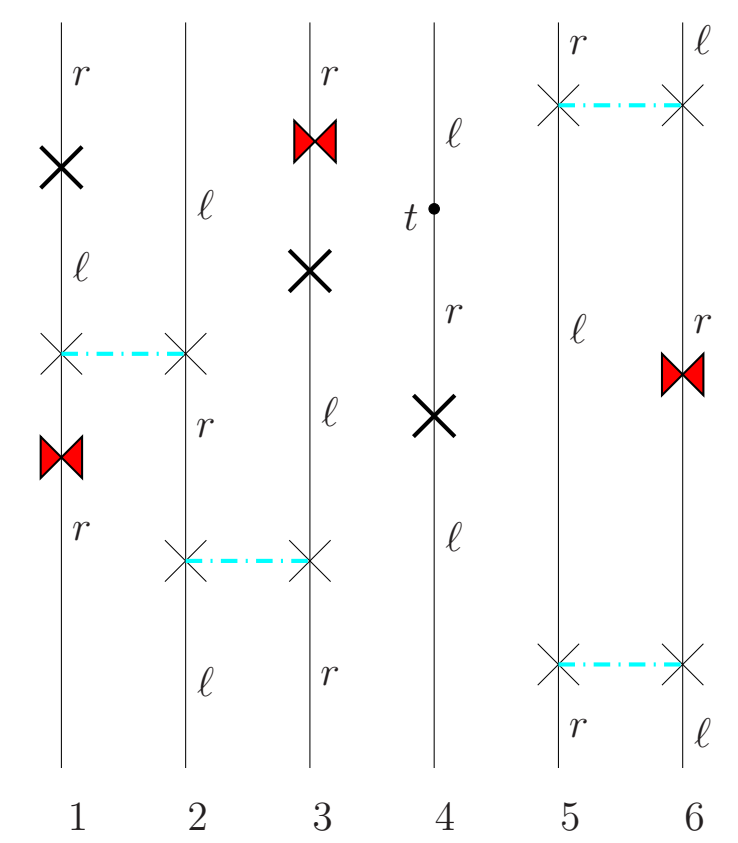

$\chi$ Arrivals of flips $\xi_{i, j} \quad \chi$ Arrivals of flips $\xi_{i \mathfrak{g}} \quad$ W Arrivals of marks $\mathfrak{m}_{i}$

Figure 1. Poisson processes of arrivals and compatible labels on $\mathfrak{S}=\cup_{1}^{6} \mathbb{S}_{\beta}^{i}: \quad(\mathrm{a}) \nu \sim(\xi, \mathfrak{m}) \quad(\mathrm{b}) \nu \stackrel{(4, t)}{\sim}(\xi, \mathfrak{m})$

For the two-point function,

$$
\left\langle\hat{\sigma}_{\mathrm{u}}^{\mathrm{z}} \hat{\sigma}_{\mathrm{v}}^{\mathrm{z}}\right\rangle=\frac{1}{\mathcal{Z}} \int \mathbb{P}(\mathrm{d} \xi, \mathrm{d} \mathfrak{m}) \sum_{\substack{u, v \\ \nu \sim(\xi, \mathfrak{m})}} \mathbf{1} .
$$

In fact, it is straightforward to check that similar formulas hold for $\mathrm{x}$-observables and mixed two-point functions (see [15] for details): Namely,

$$
\begin{aligned}
& \left\langle\hat{\Sigma}_{\mathrm{u}}^{x}\right\rangle=\frac{1}{\mathcal{Z}} \int \mathbb{P}(\mathrm{d} \xi, \mathrm{d} \mathfrak{m}) \sum_{\nu \sim(\xi, \mathfrak{m})} \mathbb{I}_{\{\nu(\mathrm{u})=\mathrm{r}\}}, \\
& \left\langle\hat{\Sigma}_{\mathrm{u}}^{x} \hat{\Sigma}_{\mathrm{v}}^{x}\right\rangle=\frac{1}{\mathcal{Z}} \int \mathbb{P}(\mathrm{d} \xi, \mathrm{d} \mathfrak{m}) \sum_{\nu \sim(\xi, \mathfrak{m})} \mathbb{I}_{\{\nu(\mathrm{u})=\mathrm{r}\}} \mathbb{I}_{\{\nu(\mathrm{v})=\mathrm{r}\}},
\end{aligned}
$$

and, for $\mathrm{u} \neq \mathrm{v}$,

$$
\left\langle\hat{\sigma}_{\mathrm{u}}^{\mathrm{z}} \hat{\Sigma}_{\mathrm{v}}^{x}\right\rangle=\frac{1}{\mathcal{Z}} \int \mathbb{P}(\mathrm{d} \xi, \mathrm{d} \mathfrak{m}) \sum_{\nu \sim \mathcal{\sim}(\xi, \mathfrak{m})} \mathbb{I}_{\{\nu(\mathrm{v})=\mathrm{r}\}} \cdot
$$


Note that once these formulas are available with $u \neq v$, they may be extended by continuity to the appropriate limiting correlation functions. We do not state them here as they will not appear in our derivations below.

Intervals, paths and replicas. Let $(\xi, \mathfrak{m})$ be a realization of the Poisson processes introduced in the previous section, $A$ a finite subset of $\mathfrak{S}$ and let $\nu$ be a compatible label $\nu \stackrel{A}{\sim}(\xi, \mathfrak{m})$. An interval of $\nu$ is a maximal connected component $I=(\mathrm{u}, \mathrm{v})$ of some $\mathbb{S}_{\beta}^{i}$ on which $\nu_{i}$ is constant. A path $\mathcal{P}$ of $(\nu, \xi, \mathfrak{m})$ is an ordered sequence $\mathfrak{I}_{1}, \mathfrak{I}_{2}, \ldots, \mathfrak{I}_{n}$, where $\mathfrak{I}_{l}$ is either an interval or a ghost site $\mathfrak{g}$ and,

(1) If $\mathfrak{I}_{l}=\left(\mathrm{u}_{l}, \mathrm{v}_{l}\right)$ and $\mathfrak{I}_{l+1}=\left(\mathrm{u}_{l+1}, \mathrm{v}_{l+1}\right)$ then either $\mathrm{v}_{l}=\mathrm{u}_{l+1}$ or $\mathrm{v}_{l}=(i, t), \mathrm{u}_{l+1}=$ $(j, t)$ and $t$ is an arrival time of $\xi_{i j}$.

(2) If $\mathfrak{I}_{l}=\left(\mathbf{u}_{l}, \mathbf{v}_{l}\right), \mathbf{v}_{l}=(j, t)$ and $\mathfrak{I}_{l+1}=\mathfrak{g}$, then $t$ is an arrival time of $\xi_{i, \mathfrak{g}}$.

(3) If $\mathfrak{I}_{l}=\mathfrak{g}, \mathfrak{I}_{l+1}=\left(\mathbf{u}_{l+1}, \mathbf{v}_{l+1}\right)$ and $\mathbf{u}_{l+1}=(j, t)$, then $t$ is an arrival time of $\xi_{j, \mathfrak{g}}$.

(4) There could not be two successive ghost sites $\mathfrak{g}$ in a path.

A path $\mathcal{P}=\left\{\mathfrak{I}_{1}, \ldots, \mathfrak{I}_{n}\right\}$ is said to be ground if it does not contain $\mathfrak{g}$, except possibly at the last step $\mathfrak{I}_{n}$. Finally, a path $\mathcal{P}$ is said to be left if all the ground intervals of $\mathcal{P}$ bear $\nu$-label $l$.

Let us define the set $\{\mathrm{u} \longleftrightarrow \mathrm{v}\}$ to be the collection of triples $(\xi, \mathfrak{m}, \nu)$ so that there exists a left path with endpoints at $u$ and $v$ and the set $\{u \stackrel{t}{\longleftrightarrow} v\}$ to be the collection of triples $(\xi, \mathfrak{m}, \nu)$ so that exists a ground left path from $u$ to $v$. Note that ground left paths are self-avoiding and that there is a unique ground left path from $\mathrm{u}$ to $\mathfrak{g}$ whenever $\nu \stackrel{\mathrm{u}}{\sim}(\xi, \mathfrak{m})$. We shall denote this path by $\mathrm{C}^{l}(\mathbf{u}, \mathfrak{g})$ and we shall use $\check{C}^{l}(\mathbf{u}, \mathfrak{g})$ for the union of its ground intervals, that is for $C^{l}(\mathbf{u}, \mathfrak{g}) \backslash \mathfrak{g}$.

Consider now two finite (and not necessarily disjoint) subsets $A, B \subset \mathfrak{S}$ and two copies $\left(\xi^{1}, \mathfrak{m}^{1}, \nu^{1}\right)$ and $\left(\xi^{2}, \mathfrak{m}^{2}, \nu^{2}\right)$ such that $\nu^{1} \stackrel{A}{\sim}\left(\xi^{1}, \mathfrak{m}^{1}\right)$ and $\nu^{2} \stackrel{B}{\sim}\left(\xi^{2}, \mathfrak{m}^{2}\right)$. We shall denote the combined processes of flips and marks as $(\eta, \mathfrak{n}) \triangleq\left(\xi^{1} \cup \xi^{2}, \mathfrak{m}^{1} \cup\right.$ $\left.\mathfrak{m}^{2}\right)$, where the union is understood in the coordinate wise sense, e.g. $\eta_{i j}=\xi_{i j}^{1} \cup$ $\xi_{i j}^{2}$. In all considerations below the processes $\left(\xi^{1}, \mathfrak{m}^{1}\right)$ and $\left(\xi^{2}, \mathfrak{m}^{2}\right)$ are independent. Consequently, $(\eta, \mathfrak{n})$ is just a collection of independent Poisson processes of arrivals with double intensities. Furthermore, given a realization $(\eta, \mathfrak{n})$, the conditional distribution of $\left(\xi^{1}, \mathfrak{m}^{1}\right) \subseteq(\eta, \mathfrak{n})$ is uniform with point mass

$$
\left(\frac{1}{2}\right)^{\#(\eta)+\#(\mathfrak{n})} \triangleq\left(\frac{1}{2}\right)^{\sum_{\mathrm{e}} \eta_{\mathrm{e}}\left[\mathbb{S}_{\beta}\right]+\sum_{i} \mathfrak{n}_{i}\left[\mathbb{S}_{\beta}\right]} .
$$

Note that given $\eta$ and the locations of the discontinuities of $\left(\nu^{1}, \nu^{2}\right)$, the arrivals of $\left(\xi^{1}, \xi^{2}\right)$ may be recovered. However it is not usually possible to reconstruct $\left(\mathfrak{m}^{1}, \mathfrak{m}^{2}\right)$ from $\mathfrak{n}$ even knowing the values of $\left(\nu^{1}, \nu^{2}\right)$.

Let us introduce geometric notions for pairs of configurations, extending our previous definitions. It will be convenient to make definitions relative to a fixed finite subset $G \subset \mathfrak{S}$. An interval $\mathfrak{I}$ of $\left(\nu^{1}, \nu^{2}\right)$ is a maximal connected component $I=(\mathrm{u}, \mathrm{v})$ of some $\mathbb{S}_{\beta}^{i}$, on which both labels $\nu^{1}$ and $\nu^{2}$ are constant and which does not contain points from $G$. A path $\mathcal{P}$ of $\left(\nu^{1}, \nu^{2}, \eta, \mathfrak{n}\right)$ is an ordered sequence $\mathfrak{I}_{1}, \mathfrak{I}_{2}, \ldots, \mathfrak{I}_{n}$, where $\mathfrak{I}_{l}$ is either an interval or a ghost site $\mathfrak{g}$ and, 
(1) If $\mathfrak{I}_{l}=\left(\mathrm{u}_{l}, \mathrm{v}_{l}\right)$ and $\mathfrak{I}_{l+1}=\left(\mathrm{u}_{l+1}, \mathrm{v}_{l+1}\right)$ then either $\mathrm{v}_{l}=\mathrm{u}_{l+1}=(i, t)$, and then either $(i, t) \in G$ or $t$ is an arrival time of $\eta_{i, \mathfrak{g}}$; or, otherwise, $\mathrm{v}_{l}=(i, t), \mathrm{u}_{l+1}=$ $(j, t)$ and $t$ is an arrival time of $\eta_{i j}$.

(2) If $\mathfrak{I}_{l}=\left(\mathbf{u}_{l}, \mathbf{v}_{l}\right), \mathbf{v}_{l}=(j, t)$ and $\mathfrak{I}_{l+1}=\mathfrak{g}$, then $t$ is an arrival time of $\eta_{j, \mathfrak{g}}$.

(3) If $\mathfrak{I}_{l}=\mathfrak{g}, \mathfrak{I}_{l+1}=\left(\mathbf{u}_{l+1}, \mathbf{v}_{l+1}\right)$ and $\mathbf{u}_{l+1}=(j, t)$, then $t$ is an arrival time of $\eta_{j, \mathfrak{g}}$.

(4) There can not be two successive ghost sites $\mathfrak{g}$ in a path.

(5) All ground intervals $\mathfrak{I}_{l} \subset \mathfrak{S}$ are disjoint.

As before, a path $\mathcal{P}=\left\{\mathfrak{I}_{1}, \ldots, \mathfrak{I}_{n}\right\}$ is said to be ground if it does not contain $\mathfrak{g}$, with a possible exception of the last step $\mathfrak{I}_{n}$. A path $\mathcal{P}=\left\{\mathfrak{I}_{1}, \mathfrak{I}_{2}, \ldots, \mathfrak{I}_{n}\right\}$ is said to be a loop if either $\mathfrak{I}_{1}=\mathfrak{I}_{n}=\mathfrak{g}$ or $\mathbf{v}_{n}=\mathbf{u}_{1}$. It is useful to keep in mind that the above notions do not depend on the values of compatible labels $\left(\nu_{1}, \nu_{2}\right)$ or arrivals of marks $\mathfrak{n}$. Rather, they only depend on the arrivals of flips $\eta$.

On the other hand, we also consider an important notion which very much depends on the pair of configurations: Let us say that the interval $\mathfrak{I}$ is blocked if (see Figure 2 ) both $\nu^{1}$ and $\nu^{2}$ equal to $r$ on $\mathfrak{I}$ and, in addition, $\mathfrak{n}(\mathfrak{I})>0$.

$>>$ Arrivals of flips and marks $\left(\xi^{1}, \mathfrak{m}^{1}\right)$ of the first replica

$<<\triangleleft$ Arrivals of flips and marks $\left(\xi^{2}, \mathfrak{m}^{2}\right)$ of the second replica

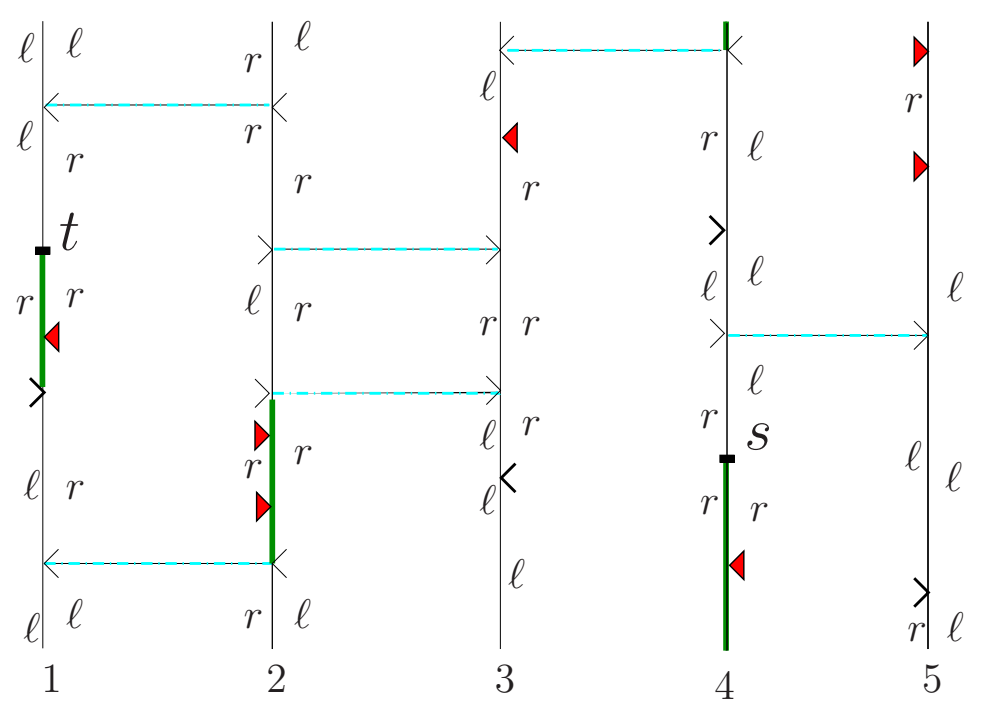

Figure 2. Special set $G=\{(1, t),(4, s)\}$ : Blocked intervals for two replicas $\left(\xi^{1}, \mathfrak{m}^{1}\right),\left(\xi^{2}, \mathfrak{m}^{2}\right)$ and two compatible labels $\nu^{1} \stackrel{(1, t)}{\sim}\left(\xi^{1}, \mathfrak{m}^{1}\right)$, $\nu^{2} \stackrel{(4, s)}{\sim}\left(\xi^{2}, \mathfrak{m}^{2}\right)$

A path $\mathcal{P}=\left\{\mathfrak{I}_{1}, \ldots, \mathfrak{I}_{n}\right\}$ is said to be unblocked if it does not contain blocked intervals. We shall say that $\{\mathrm{u} \stackrel{*}{\longleftrightarrow} \mathrm{v}\}$ if, for $G=\{\mathrm{u}, \mathrm{v}\}$, there exists an unblocked path with end-points at $u$ and $v$, and we shall write $\{u \stackrel{* t}{\longleftrightarrow} v\}$ whenever there exists a ground unblocked path from $u$ to $v$. 
Basic Transformation. Let $\mathcal{P}=\left(\mathfrak{I}_{1}, \ldots, \mathfrak{I}_{n}\right)$ be an unblocked path of $\left(\nu^{1}, \nu^{2}, \eta, \mathfrak{n}\right)$ from $\mathrm{u}$ to $\mathrm{v}$. Obviously the labels $\nu^{1}$ and $\nu^{2}$ unambiguously define the splitting $\eta=\xi^{1} \cup \xi^{2}$. Moreover, since $\mathcal{P}$ is unblocked, $\nu^{1}$ and $\nu^{2}$ unambiguously define the splitting of marks $\mathfrak{n}=\mathfrak{m}^{1} \cup \mathfrak{m}^{2}$ along $\mathcal{P}$.

Make the following transformation of labels and marks on each of the ground intervals $\mathfrak{I}$ of $\mathcal{P}$ :

(1) If the $\left(\nu^{1}, \nu^{2}\right)$ label of $\mathfrak{I}$ is $(l, r)$, then flip it to $(r, l)$ and transfer all marks accordingly - set $\mathfrak{m}^{1}(\mathfrak{I})=\mathfrak{m}^{2}(\mathfrak{I})$ and set $\mathfrak{m}^{2}(\mathfrak{I})=0$. Perform the analogous procedure if the label is $(r, l)$.

(2) If the label is $(l, l)$ then flip it to $(r, r)$. Accordingly, if the label is $(r, r)$, then flip it to $(l, l)$. Note that in the latter case, since we are moving along an unblocked path, $\mathfrak{n}(\mathfrak{I})$ has to be equal to zero, and no incompatibility arises.

(3) Adjust $\xi^{1}$ and $\xi^{2}$ accordingly - those are, of course completely defined by the labels (flips of the labels, to be precise).

The above transformation, let us call it $\Phi_{\mathcal{P}}$, defines a map

$$
\left\{\left(\nu^{1}, \xi^{1}, \mathfrak{m}^{1}\right),\left(\nu^{2}, \xi^{2}, \mathfrak{m}^{2}\right)\right\} \mapsto\left\{\left(\widetilde{\nu}^{1}, \widetilde{\xi}^{1}, \widetilde{\mathfrak{m}}^{1}\right),\left(\widetilde{\nu}^{2}, \widetilde{\xi}^{2}, \widetilde{\mathfrak{m}}^{2}\right)\right\}
$$

The map $\Phi_{\mathcal{P}}$ enjoys the following set of properties:

(1) It is invertible: Indeed just apply $\Phi_{\mathcal{P}}$ once more to recover the original data.

(2) It does not change $\nu^{1}$ and $\nu^{2}$ labels and $\mathfrak{m}^{1}, \mathfrak{m}^{2}$-marks on intervals which do not belong to $\mathcal{P}$. In addition, the original and modified configurations have the same set of intervals (defined by $\eta$, u and $v$ ), and $\Phi_{\mathcal{P}}$ does not change the blocked/unblocked status of any of those.

(3) If $\nu^{1} \stackrel{A}{\sim}\left(\xi^{1}, \mathfrak{m}^{1}\right)$ and $\nu^{2} \stackrel{B}{\sim}\left(\xi^{2}, \mathfrak{m}^{2}\right)$, then

$$
\widetilde{\nu}^{1} \stackrel{A \Delta\{u, v\}}{\sim}\left(\widetilde{\xi}^{1}, \widetilde{\mathfrak{m}}^{1}\right) \text { and } \widetilde{\nu}^{2} \stackrel{B \Delta\{u, v\}}{\sim}\left(\widetilde{\xi}^{2}, \widetilde{\mathfrak{m}}^{2}\right) .
$$

(4) It is measure preserving: In view of $(\underline{2.6}),\left(\xi^{1}, \mathfrak{m}^{1}\right)$ and $\left(\widetilde{\xi}^{1}, \widetilde{\mathfrak{m}}^{1}\right)$ have the same conditional weights.

Minimal paths. Most of the transformations we are going to perform will be along minimal unblocked paths, often satisfying additional geometric constraints. Let us, therefore, define what we mean by minimal. First of all given an unblocked path $\mathcal{P}=\left(\mathfrak{I}_{1}, \ldots, \mathfrak{I}_{n}\right)$ define its length as $|\mathcal{P}| \triangleq \sum_{1}^{n}\left|\mathfrak{I}_{l}\right|$, where $|\mathfrak{I}|$ is the Euclidean length if $\mathfrak{I}$ is a ground interval, and, by definition, $|\mathfrak{g}|=0$. Consider now two replicas $\left(\xi^{1}, \mathfrak{m}^{1}\right),\left(\xi^{2}, \mathfrak{m}^{2}\right)$ and a pair of compatible labels $\nu^{1}, \nu^{2}$. Let $\mathbf{u}, \mathbf{v} \in \mathfrak{S} \cup \mathfrak{g}$ and assume that there are unblocked paths from $u$ to $v$. Then the minimal path $\mathrm{C}^{*}(\mathrm{u}, \mathrm{v})$ satisfies,

$$
\left|\mathrm{C}^{*}(\mathrm{u}, \mathrm{v})\right| \leq|\mathcal{P}| \text { for any unblocked path } \mathcal{P} \text { from } \mathrm{u} \text { to } \mathrm{v} .
$$

It is easy to see that in general (2.8) alone does not define $\mathrm{C}^{*}(\mathrm{u}, \mathrm{v})$ uniquely, and one needs to impose an additional rule in order to chose the minimal path from a set of paths with the same minimal length. For example the following rule will do: Write a coarse grained description of $\mathcal{P}(\mathrm{u}, \mathrm{v})=\mathfrak{R}_{1}, \ldots, \mathfrak{R}_{m}$, where $\mathfrak{R}_{l}$ is either a ghost site $\mathfrak{g}$ or a maximal collection of successive ground intervals of $\mathcal{P}$ on some $\mathbb{S}_{\beta}^{i}$. 
Then for two unblocked paths $\mathcal{P}=\left(\mathfrak{R}_{1}, \ldots \mathfrak{R}_{m}\right)$ and $\mathcal{P}^{\prime}=\left(\mathfrak{R}_{1}^{\prime}, \ldots, \mathfrak{R}_{k}^{\prime}\right)$ we shall say that $\mathcal{P} \prec \mathcal{P}^{\prime}$ if either $|\mathcal{P}|<\left|\mathcal{P}^{\prime}\right|$, or if the lengths are equal, there exists $l$ such that

$$
\left|\mathfrak{R}_{i}\right|=\left|\mathfrak{R}_{i}^{\prime}\right| \text { for } i=1, \ldots, l-1 \text {, but }\left|\mathfrak{R}_{l}\right|>\left|\mathfrak{R}_{l}^{\prime}\right| \text {. }
$$

Then $\mathrm{C}^{*}(\mathrm{u}, \mathrm{v})$ is unambiguously defines as the unique unblocked path from $\mathrm{u}$ to $\mathrm{v}$ which is $\prec$-less than any other unblocked path from $u$ to $v$. In other words, the minimal path, as we define it, is the most conservative of all the paths of the same minimal length: it tries to stay as much as possible on each subsequent spatial circle $\mathbb{S}_{\beta}$

The important feature of the path transformation $\Phi$ which was introduced above is (see Figure 3): If $\mathrm{C}^{*}(\mathbf{u}, \mathbf{v})$ is the minimal path, then it remains so after $\Phi_{\mathrm{C}^{*}(\mathbf{u}, \mathrm{v})}$ is performed. As a result, transformations along minimal paths are well defined and invertible.

\section{SWitching LEMmas AND RELATED CORRELATION INEQUALities}

Recall that $\left(\xi^{1}, \mathfrak{m}^{1}\right)$ and $\left(\xi^{2}, \mathfrak{m}^{2}\right)$ are independent copies of our Poisson processes of flips and marks, and that we use $\eta=\xi^{1} \cup \xi^{2}, \mathfrak{n}=\mathfrak{m}^{1} \cup \mathfrak{m}^{2}$ for the combined processes. Let $\mathbb{E}$ denote the expectation with respect to two independent replicas of Poisson processes of flips and marks; $\left(\xi^{1}, \mathfrak{m}^{1}\right)$ and $\left(\xi^{2}, \mathfrak{m}^{2}\right)$. In this section, we give exact formulae for the truncated correlations appearing in (1.2) and discuss the term $\partial M / \partial \rho$ which appears in Theorem B.

Representation of $\left\langle\hat{\sigma}_{\mathrm{u}}^{z} ; \hat{\sigma}_{\mathrm{v}}^{\mathrm{z}}\right\rangle$. In view of (2.6) we can record (2.2) in terms of two replicas as,

$$
\left\langle\hat{\sigma}_{\mathrm{u}}^{\mathrm{z}}\right\rangle\left\langle\hat{\sigma}_{\mathrm{v}}^{\mathrm{z}}\right\rangle=\frac{1}{\mathcal{Z}^{2}} \int \mathbb{P}(\mathrm{d} \eta, \mathrm{d} \mathfrak{n})\left(\frac{1}{2}\right)^{\#(\eta)+\#(\mathfrak{n})} \sum_{\substack{\xi^{1} \cup \xi^{2}=\eta \\ \mathfrak{m}^{1} \cup \mathfrak{m}^{2}=\mathfrak{n}}} \sum_{\substack{\nu^{1} \stackrel{\sim}{\sim}\left(\xi^{1}, \mathfrak{m}^{2}\right) \\ \nu^{2} \sim\left(\xi^{1}, \mathfrak{m}^{2}\right)}} \mathbf{1}
$$

Similarly, we can record (2.1) and (2.3) as,

$$
\left\langle\hat{\sigma}_{\mathrm{u}}^{\mathrm{z}} \hat{\sigma}_{\mathrm{v}}^{\mathrm{z}}\right\rangle=\frac{\mathcal{Z}\left\langle\hat{\sigma}_{i}^{\mathrm{z}} \hat{\sigma}_{j}^{\mathrm{z}}\right\rangle_{\Lambda}}{\mathcal{Z}}=\frac{1}{\mathcal{Z}^{2}} \int \mathbb{P}(\mathrm{d} \eta, \mathrm{d} \mathfrak{n})\left(\frac{1}{2}\right)^{\#(\eta)+\#(\mathfrak{n})} \sum_{\substack{\xi^{1} \cup \xi^{2}=\eta \\ \mathfrak{m}^{1} \cup \mathfrak{m}^{2}=\mathfrak{n}}} \sum_{\substack{\nu^{1} \sim\left(\xi^{1}, \mathfrak{m}^{1}\right) \\ \nu^{2} \sim \mathcal{L}^{2}}} 1 .
$$

Let us have a closer look at (3.1). The constraint $\nu^{1} \stackrel{u}{\sim}\left(\xi^{1}, \mathfrak{m}^{1}\right)$ implies that there is a path $\mathcal{P}$ from $\mathrm{u}$ to $\mathfrak{g}$ such that $\nu^{1} \equiv l$ on $\mathcal{P}$. In particular this path $\mathcal{P}$ must be unblocked. An analogous statement also applies with respect to $\mathrm{v}$ in the second replica. Therefore, one can rewrite (3.1) as

$$
\begin{aligned}
& \left\langle\hat{\sigma}_{\mathrm{u}}^{\mathrm{z}}\right\rangle\left\langle\hat{\sigma}_{\mathrm{v}}^{\mathrm{z}}\right\rangle \\
& =\frac{1}{\mathcal{Z}^{2}} \int \mathbb{P}(\mathrm{d} \eta, \mathrm{d} \mathfrak{n})\left(\frac{1}{2}\right)^{\#(\eta)+\#(\mathfrak{n})} \\
& \quad \times \sum_{\substack{\xi^{1} \cup \xi^{2}=\eta \\
\mathfrak{m}^{1} \cup \mathfrak{m}^{2}=\mathfrak{n}}} \sum_{\substack{\nu^{1} \stackrel{u}{\sim}\left(\xi^{1}, \mathfrak{m}^{1}\right) \\
\nu^{2} \sim\left(\xi^{2}, \mathfrak{m}^{2}\right)}} \mathbb{I}_{\{\mathrm{u} \stackrel{*}{\sim} \mathfrak{g}\}} \mathbb{I}_{\{\mathfrak{v} \stackrel{*}{\longleftrightarrow} \mathfrak{g}\}} .
\end{aligned}
$$


NICHOLAS CRAWFORD AND DMITRY IOFFE

(a)

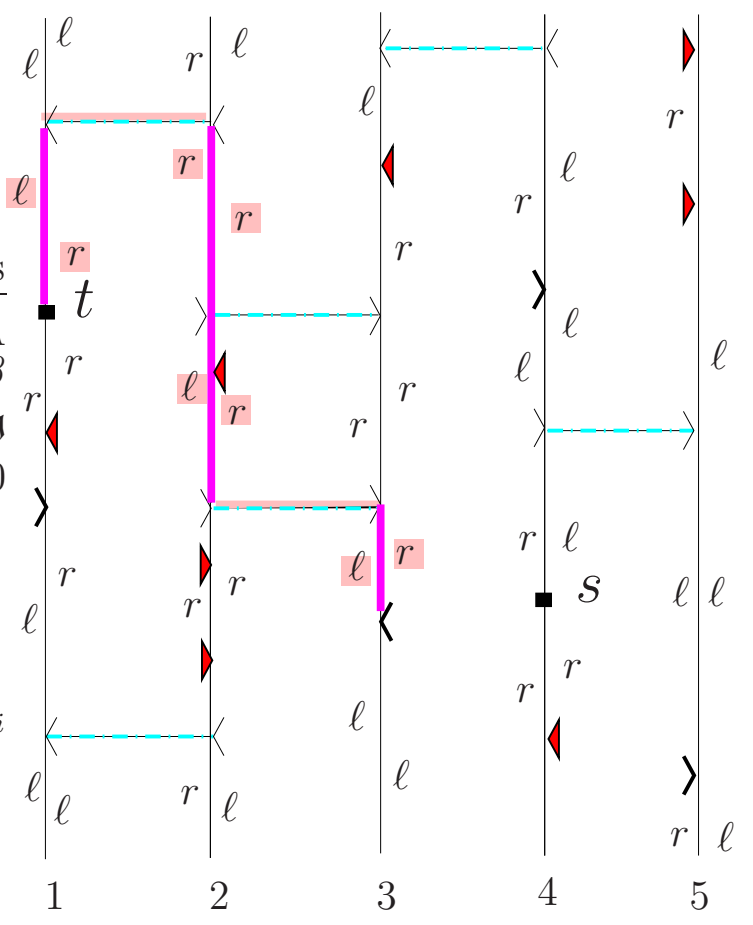

(b)

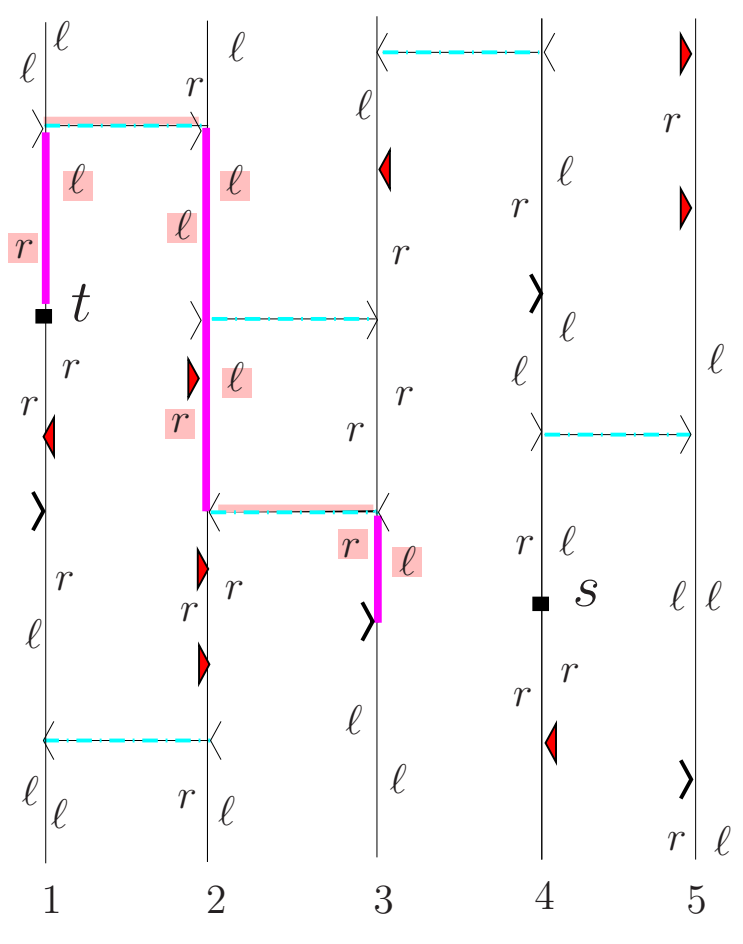

$>>\quad$ Arrivals of flips and marks $\left(\xi^{1}, \mathfrak{m}^{1}\right)$ of the first replica

$<<\triangleleft$ Arrivals of flips and marks $\left(\xi^{2}, \mathfrak{m}^{2}\right)$ of the second replica

Figure 3. Two replicas $\left(\xi^{1}, \mathfrak{m}^{1}\right),\left(\xi^{2}, \mathfrak{m}^{2}\right)$ and two compatible labels $\nu^{1} \stackrel{u}{\sim}\left(\xi^{1}, \mathfrak{m}^{1}\right), \nu^{2} \stackrel{v}{\sim}\left(\xi^{2}, \mathfrak{m}^{2}\right)$, where $\mathrm{u}=(1, t)$ and $\mathbf{v}=(4, s)$.

(a) Minimal unblocked path $\mathrm{C}^{*}(\mathrm{u}, \mathrm{v})$ from $\mathrm{u}$ to the ghost site $\mathfrak{g}$.

(b) Basic transformation: New labels $\widetilde{\nu}^{1} \sim\left(\widetilde{\xi}^{1}, \widetilde{\mathfrak{m}}^{1}\right)$ and $\widetilde{\nu}^{2} \stackrel{\text { u,v }}{\sim}\left(\widetilde{\xi}^{1}, \widetilde{\mathfrak{m}}^{1}\right)$.

Labels which are switched along the minimal path are shaded. Note that the flips and the marks are switched accordingly.

Similarly, one can rewrite (3.2) as,

$$
\begin{aligned}
& \left\langle\hat{\sigma}_{\mathrm{u}}^{z} \hat{\sigma}_{\mathrm{v}}^{z}\right\rangle \\
& =\frac{1}{\mathcal{Z}^{2}} \int \mathbb{P}(\mathrm{d} \eta, \mathrm{d} \mathfrak{n})\left(\frac{1}{2}\right)^{\#(\eta)+\#(\mathfrak{n})} \times \sum_{\substack{\xi^{1} \cup \xi^{2}=\eta \\
\mathfrak{m}^{1} \cup \mathfrak{m}^{2}=\mathfrak{n}}} \sum_{\substack{\nu 1 \sim\left(\xi^{1}, \mathfrak{m}^{1}\right) \\
\nu^{2} \sim \sim^{2}\left(\xi^{2}, \mathfrak{m}^{2}\right)}} \mathbb{1}_{\left.\left\{\mathrm{u} \longleftrightarrow^{*}\right) v\right\}} .
\end{aligned}
$$

Let us fix a realization of $(\eta, \mathfrak{n})$. Define $\mathcal{A}_{\mathrm{u}, \mathrm{v}}^{\mathfrak{g}}=\mathcal{A}_{\mathrm{u}, \mathrm{v}}^{\mathfrak{g}}(\eta, \mathfrak{n})$ to be the set of pairs of objects $\left\{\left(\nu^{1}, \xi^{1}, \mathfrak{m}^{1}\right),\left(\nu^{2}, \xi^{2}, \mathfrak{m}^{2}\right)\right\}$ which contribute to the double sum on the right hand side of (3.3). Similarly let $\mathcal{A}_{\mathrm{u}, \mathrm{v}}$ be the set of pairs of objects (currents and labels) which contribute to the double sum on the right hand side of (3.4). Each of 
the objects in $\mathcal{A}_{\mathrm{u}, \mathrm{v}}^{\mathfrak{g}}$ contains an unblocked path, and hence the minimal unblocked path $\mathrm{C}^{*}(\mathrm{u}, \mathfrak{g})$ from $\mathrm{u}$ to $\mathfrak{g}$. We claim that the map $, \Phi \equiv \Phi_{\mathrm{C}^{*}(\mathrm{u}, \mathfrak{g})}: \mathcal{A}_{\mathrm{u}, \mathrm{v}}^{\mathfrak{g}} \mapsto \mathcal{A}_{\mathrm{u}, \mathrm{v}}$ is a measure preserving injection. This follows immediately from the properties of basic transformations and minimal paths. However, $\Phi$ is not onto: any couple of objects in the image $\left\{\left(\nu^{1}, \xi^{1}, \mathfrak{m}^{1}\right),\left(\nu^{2}, \xi^{2}, \mathfrak{m}^{2}\right)\right\} \in \Phi\left(\mathcal{A}_{\mathrm{u}, \mathrm{v}}^{\mathfrak{g}}\right)$ necessarily contains an unblocked path from $u$ to $\mathfrak{g}$. We have proved:

Theorem 3.1. Truncated z-correlation functions satisfy the following version of the Switching Lemma:

$$
\begin{aligned}
& \left\langle\hat{\sigma}_{\mathrm{u}}^{\mathrm{z}} ; \hat{\sigma}_{\mathrm{v}}^{\mathrm{z}}\right\rangle
\end{aligned}
$$

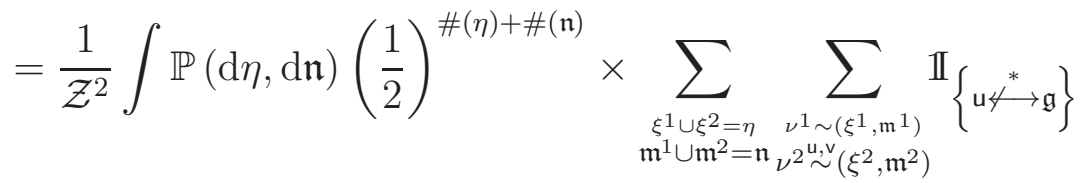

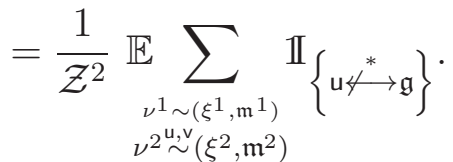

Representation of $\left\langle\hat{\Sigma}_{\mathrm{u}}^{x} ; \hat{\Sigma}_{\mathrm{v}}^{x}\right\rangle$. Consider two independent replicas $\left(\xi^{1}, \mathfrak{m}^{1}\right),\left(\xi^{2}, \mathfrak{m}^{2}\right)$ and two labels $\nu^{1} \sim\left(\xi^{1}, \mathfrak{m}^{1}\right)$ and $\nu^{2} \sim\left(\xi^{2}, \mathfrak{m}^{2}\right)$. Let us say that a couple of labels $\left(\nu^{1}, \nu^{2}\right) \in\left[(r, r)_{\mathrm{u}},(r, l)_{\mathrm{v}}\right]$ if $\nu^{1}(\mathrm{u})=r=\nu^{1}(\mathrm{v}), \nu^{2}(\mathrm{u})=r$ and $\nu^{2}(\mathrm{v})=l$. The events, $\left\{\left(\nu^{1}, \nu^{2}\right) \in\left[(r, l)_{\mathrm{u}},(r, l)_{\mathrm{v}}\right]\right\},\left\{\left(\nu^{1}, \nu^{2}\right) \in\left[(l, l)_{\mathrm{u}},(r, l)_{\mathrm{v}}\right]\right\}$ etc. (all together 16 events) are defined in a completely similar fashion. In terms of two replicas, the representation formulas (2.4) read as,

$$
\begin{aligned}
\left\langle\hat{\Sigma}_{\mathrm{u}}^{x} \hat{\Sigma}_{\mathrm{v}}^{x}\right\rangle=\frac{1}{\mathcal{Z}^{2}} \mathbb{E} \sum_{\substack{\nu^{1} \sim\left(\xi^{1}, \mathfrak{m}^{1}\right) \\
\nu^{2} \sim\left(\xi^{2}, \mathfrak{m}^{2}\right)}}( & \mathbb{I}_{\left\{\left(\nu^{1}, \nu^{2}\right) \in\left[(\mathrm{r}, \mathrm{r})_{\mathrm{u}},(\mathrm{r}, \mathrm{r})_{\mathrm{v}}\right]\right\}}+\mathbb{I}_{\left\{\left(\nu^{1}, \nu^{2}\right) \in\left[(\mathrm{r}, \mathrm{r})_{\mathrm{u}},(\mathrm{r}, \mathrm{l})_{\mathrm{v}}\right]\right\}} \\
& \left.+\mathbb{I}_{\left\{\left(\nu^{1}, \nu^{2}\right) \in\left[(\mathrm{r}, \mathrm{l})_{\mathrm{u}},(\mathrm{r}, \mathrm{r})_{\mathrm{v}}\right]\right\}}+\mathbb{I}_{\left\{\left(\nu^{1}, \nu^{2}\right) \in\left[(\mathrm{r}, \mathrm{l})_{\mathrm{u}},(\mathrm{r}, \mathrm{l})_{\mathrm{v}}\right]\right\}}\right) .
\end{aligned}
$$

Similarly,

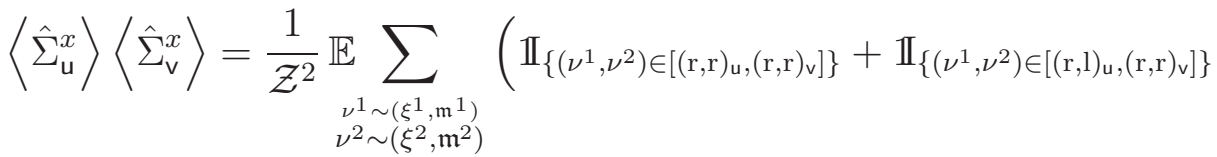

$$
\begin{aligned}
& \left.+\mathbb{1}_{\left\{\left(\nu^{1}, \nu^{2}\right) \in\left[(\mathrm{r}, \mathrm{r})_{\mathrm{u}},(1, \mathrm{r})_{\mathrm{v}}\right]\right\}}+\mathbb{1}_{\left\{\left(\nu^{1}, \nu^{2}\right) \in\left[(\mathrm{r}, \mathrm{l})_{\mathrm{u}},(1, \mathrm{r})_{\mathrm{v}}\right]\right\}}\right) .
\end{aligned}
$$

Evidently,

$$
\mathbb{E} \sum_{\substack{\nu^{1} \sim\left(\xi^{1}, \mathfrak{m}^{1}\right) \\ \nu^{2} \sim\left(\xi^{2}, \mathfrak{m}^{2}\right)}} \mathbb{I}_{\left\{\left(\nu^{1}, \nu^{2}\right) \in\left[(\mathrm{r}, \mathrm{r})_{\mathrm{u}},(\mathrm{r}, \mathrm{l}) \mathrm{v}\right]\right\}}=\mathbb{E}_{\substack{\nu^{1} \sim\left(\xi^{1}, \mathfrak{m}^{1}\right) \\ \nu^{2} \sim\left(\xi^{2}, \mathfrak{m}^{2}\right)}} \mathbb{I}_{\left\{\left(\nu^{1}, \nu^{2}\right) \in\left[(\mathrm{r}, \mathrm{r})_{\mathrm{u}},(1, \mathrm{r})_{\mathrm{v}}\right]\right\}}
$$


Consequently, we arrive to the following representation for the truncated two point function:

$$
\left\langle\hat{\Sigma}_{\mathrm{u}}^{x} ; \hat{\Sigma}_{\mathrm{v}}^{x}\right\rangle=\frac{1}{\mathcal{Z}^{2}} \mathbb{\substack { \nu ^ { 1 } \sim ( \xi ^ { 1 } , \mathfrak { m } ^ { 1 } ) \\ \nu ^ { 2 } \sim ( \xi ^ { 2 } , \mathfrak { m } ^ { 2 } ) }}\left(\mathbb{1}_{\left\{\left(\nu^{1}, \nu^{2}\right) \in\left[(\mathrm{r}, 1)_{\mathrm{u}},(\mathrm{r}, 1)_{\mathrm{v}}\right]\right\}}-\mathbb{1}_{\left\{\left(\nu^{1}, \nu^{2}\right) \in\left[(\mathrm{r}, \mathrm{l})_{\mathrm{u}},(1, \mathrm{r})_{\mathrm{v}}\right]\right\}}\right) .
$$

At this stage we proceed much along the lines of our proof of Theorem 3.1. Fix a realization of $(\eta, \mathfrak{n})$ and let $\mathcal{B}_{+}(\eta, \mathfrak{n})$ be the set of pairs of objects $\left\{\left(\nu^{1}, \xi^{1}, \mathfrak{m}^{1}\right),\left(\nu^{2}, \xi^{2}, \mathfrak{m}^{2}\right)\right\}$ which contribute to the sum

$$
\sum_{\substack{\xi^{1} \cup \xi^{2}=\eta \\ \mathfrak{m}^{1} \cup \mathfrak{m}^{2}=\mathfrak{n}}} \sum_{\substack{\nu^{1} \sim\left(\xi^{1}, \mathfrak{m}^{1}\right) \\ \nu^{2} \sim\left(\xi^{2}, \mathfrak{m}^{2}\right)}} \mathbb{1}_{\left\{\left(\nu^{1}, \nu^{2}\right) \in\left[(\mathrm{r}, 1)_{\mathrm{u}},(\mathrm{r}, \mathrm{l}) \mathrm{v}\right]\right\}}
$$

Similarly, let $\mathcal{B}_{-}(\eta, \mathfrak{n})$ be the set of pairs of objects $\left\{\left(\nu^{1}, \xi^{1}, \mathfrak{m}^{1}\right),\left(\nu^{2}, \xi^{2}, \mathfrak{m}^{2}\right)\right\}$ which contribute to the sum

$$
\sum_{\substack{\xi^{1} \cup \xi^{2}=\eta \\ \mathfrak{m}^{1} \cup \mathfrak{m}^{2}=\mathfrak{n}}} \sum_{\substack{\nu 1 \sim\left(\xi^{1}, \mathfrak{m}^{1}\right) \\ \nu^{2} \sim\left(\xi^{2}, \mathfrak{m}^{2}\right)}} \mathbb{1}_{\left\{\left(\nu^{1}, \nu^{2}\right) \in\left[(\mathrm{r}, 1)_{\mathrm{u}},(1, \mathrm{r})_{\mathfrak{v}}\right]\right\}}
$$

An injective map $\Psi=\Psi_{\eta, \mathfrak{n}}: \mathcal{B}_{-}(\eta, \mathfrak{n}) \mapsto \mathcal{B}_{+}(\eta, \mathfrak{n})$ is constructed as follows: Any

$$
\left\{\left(\nu^{1}, \xi^{1}, \mathfrak{m}^{1}\right),\left(\nu^{2}, \xi^{2}, \mathfrak{m}^{2}\right)\right\} \in \mathcal{B}_{-}(\eta, \mathfrak{n})
$$

contains an unblocked loop $\mathcal{L}$ from $v$ to $v$ such that $u \notin \mathcal{L}$. Indeed, such a loop may be constructed with $\nu^{1} \equiv l$. Now just choose the minimal such loop (in the sense discussed above) and perform on this minimal loop the very same surgery as in the Basic Transformation. Again, the property that the loop is minimal is not changed under the surgery and hence $\Psi$ is invertible. On the other hand, the image set $\Psi \mathcal{B}_{-}(\eta, \mathfrak{n}) \subset \mathcal{B}_{+}(\eta, \mathfrak{n})$.

Geometrically, it is evident that $\mathcal{B}_{+} \backslash \Psi \mathcal{B}_{-}$is characterized by the following condition: A pair $\left\{\left(\nu^{1}, \xi^{1}, \mathfrak{m}^{1}\right),\left(\nu^{2}, \xi^{2}, \mathfrak{m}^{2}\right)\right\}$ from $\mathcal{B}_{+}$belongs to $\mathcal{B}_{+} \backslash \Psi \mathcal{B}_{-}$if and only if any unblocked loop containing $v$ also contains $u$. In this case, let us say that $u$ is loop-pivotal for $v$.

We conclude:

Theorem 3.2. Truncated $\mathrm{x}$-correlation functions satisfy the following version of the Switching Lemma:

$$
\left\langle\hat{\Sigma}_{\mathrm{u}}^{x} ; \hat{\Sigma}_{\mathrm{v}}^{x}\right\rangle=\frac{1}{\mathcal{Z}^{2}} \underset{\substack{\nu^{1} \sim\left(\xi^{1}, \mathfrak{m}^{1}\right) \\ \nu^{2} \sim\left(\xi^{2}, \mathfrak{m}^{2}\right)}}{\mathbb{E}} \mathbb{I}_{\left\{\left(\nu^{1}, \nu^{2}\right) \in\left[(\mathrm{r}, 1)_{\mathrm{u}},(\mathrm{r}, \mathrm{l}) \mathrm{v}\right]\right\}} \mathbb{I}_{\{\mathrm{v} \text { is loop pivotal for } \mathrm{u}\}} .
$$

Representation of cross-correlations. As before, let $\mathbb{E}$ denote the expectation with respect to two independent replicas of Poisson processes of flips and marks; $\left(\xi^{1}, \mathfrak{m}^{1}\right)$ and $\left(\xi^{2}, \mathfrak{m}^{2}\right)$. With this notation we have (from (2.2) , the first of (2.4) and (2.5) ),

$$
\left\langle\hat{\sigma}_{\mathrm{u}}^{z} \hat{\Sigma}_{\mathrm{v}}^{x}\right\rangle=\frac{1}{\mathcal{Z}^{2}} \mathbb{E} \sum_{\substack{\nu^{1} \stackrel{u}{\sim}\left(\xi^{1}, \mathfrak{m}^{1}\right) \\ \nu^{2} \sim\left(\xi^{2}, \mathfrak{m}^{2}\right)}} \mathbb{1}_{\left\{\nu^{1}(\mathrm{v})=\mathrm{r}\right\}}
$$


and, accordingly,

$$
\left\langle\hat{\sigma}_{\mathrm{u}}^{\mathrm{z}}\right\rangle\left\langle\hat{\Sigma}_{\mathrm{v}}^{x}\right\rangle=\frac{1}{\mathcal{Z}^{2}} \mathbb{E} \sum_{\substack{\nu^{1} \stackrel{u}{\sim}\left(\xi^{1}, \mathfrak{m}^{1}\right) \\ \nu^{2} \sim\left(\xi^{2}, \mathfrak{m}^{2}\right)}} \mathbb{1}_{\left\{\nu^{2}(\mathrm{v})=\mathrm{r}\right\}} \cdot
$$

Fix a realization of $(\eta, \mathfrak{n})$ and let $\mathcal{D}_{+}(\eta, \mathfrak{n})$ be the set of pairs of objects $\left\{\left(\nu^{1}, \xi^{1}, \mathfrak{m}^{1}\right),\left(\nu^{2}, \xi^{2}, \mathfrak{m}^{2}\right)\right\}$ which contribute to the sum

$$
\sum_{\substack{\xi^{1} \cup \xi^{2}=\eta \\ \mathfrak{m}^{1} \cup \mathfrak{m}^{2}=\mathfrak{n}}} \sum_{\substack{\nu^{1} \sim \\ \nu^{2} \sim\left(\xi^{1}, \mathfrak{m}^{1}\right) \\ \nu^{2} \sim\left(\xi^{2}, \mathfrak{m}^{2}\right)}} \mathbb{I}_{\left\{\nu^{2}(\mathrm{v})=\mathrm{r}\right\}}
$$

Similarly, let $\mathcal{D}_{-}(\eta, \mathfrak{n})$ be the set of pairs of objects $\left\{\left(\nu^{1}, \xi^{1}, \mathfrak{m}^{1}\right),\left(\xi^{2}, \mathfrak{m}^{2}, \nu^{2}\right)\right\}$ which contribute to the sum

$$
\sum_{\substack{\xi^{1} \cup \xi^{2}=\eta \\ \mathfrak{m}^{1} \cup \mathfrak{m}^{2}=\mathfrak{n}}} \sum_{\substack{\nu^{1} \stackrel{u}{\sim}\left(\xi^{1}, \mathfrak{m}^{1}\right) \\ \nu^{2} \sim\left(\xi^{2}, \mathfrak{m}^{2}\right)}} \mathbb{I}_{\left\{\nu^{1}(\mathrm{v})=\mathrm{r}\right\}}
$$

Note now that any pair of objects $\left\{\left(\nu^{1}, \xi^{1}, \mathfrak{m}^{1}\right),\left(\nu^{2}, \xi^{2}, \mathfrak{m}^{2}\right)\right\} \in \mathcal{D}_{-}$contains an unblocked and hence the minimal unblocked path $\mathrm{C}^{*, \gamma}(\mathbf{u}, \mathfrak{g})$ from $\mathrm{u}$ to $\mathfrak{g}$ which avoids v. An injective map $\Omega=\Omega_{\eta, \mathfrak{n}}: \mathcal{D}_{-}(\eta, \mathfrak{n}) \mapsto \mathcal{D}_{+}(\eta, \mathfrak{n})$ is then constructed as follows:

(1) Perform the Basic Transformation along the minimal path $\mathrm{C}^{*, \boldsymbol{y}}(\mathbf{u}, \mathfrak{g})$.

(2) Using the symmetry of replicas, rename the resulting

$$
\left(\widetilde{\nu}^{1}, \widetilde{\xi}^{1}, \widetilde{\mathfrak{m}}^{1}\right) \leftrightarrow\left(\widetilde{\nu}^{2}, \widetilde{\xi}^{2}, \widetilde{\mathfrak{m}}^{2}\right)
$$

It is evident that $\mathcal{D}_{+} \backslash \Omega \mathcal{D}_{-}$is characterized by the following condition: A pair of objects $\left\{\left(\nu^{1}, \xi^{1}, \mathfrak{m}^{1}\right),\left(\nu^{2}, \xi^{2}, \mathfrak{m}^{2}\right)\right\}$ from $\mathcal{D}_{+}$belongs to $\mathcal{D}_{+} \backslash \Omega \mathcal{D}_{-}$if and only if any unblocked path from $\mathrm{u}$ to the ghost site $\mathfrak{g}$ contains $\mathrm{v}$. Let us say that $\mathrm{v}$ is pivotal for $\mathrm{u} \stackrel{*}{\longleftrightarrow} \mathfrak{g}$ if the latter condition holds. We have proved:

Theorem 3.3. Truncated cross-correlation functions satisfy the following version of the Switching Lemma:

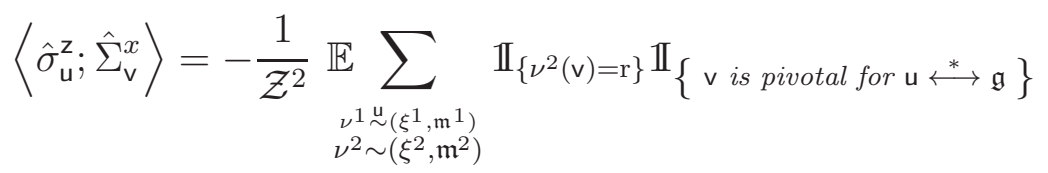

Note that the following (straightforward) generalization of Theorem 3.3 holds. Let $G=\left\{\mathrm{v}_{1}, \ldots, \mathrm{v}_{l}, \mathrm{v}_{l}, \ldots, \mathrm{v}_{l+k}\right\}$ be a finite subset of $\mathfrak{S}$ which is time-ordered in the following sense: The coordinates $\mathrm{v}_{q}=\left(1_{q}, t_{q}\right)$ satisfy $t_{q}<t_{p}$ whenever $q<p$. Let $\mathrm{u}=(i, t)$ be such that $t_{l}<t<t_{l+1}$. Then, the truncated cross-correlation $\left\langle\hat{\sigma}_{\mathrm{u}}^{\mathrm{z}} ; \prod_{1}^{l+k} \hat{\Sigma}_{\mathrm{v}_{q}}^{x}\right\rangle$ is defined as

$$
\left\langle\hat{\sigma}_{\mathrm{u}}^{\mathrm{z}} ; \prod_{1}^{l+k} \hat{\Sigma}_{\mathrm{v}_{q}}^{x}\right\rangle=\left\langle\prod_{1}^{l} \hat{\Sigma}_{\mathrm{v}_{q}}^{x} \hat{\sigma}_{\mathrm{u}}^{\mathrm{z}} \prod_{l+1}^{l+k} \hat{\Sigma}_{\mathrm{v}_{q}}^{x}\right\rangle-\left\langle\hat{\sigma}_{\mathrm{u}}^{\mathrm{z}}\right\rangle\left\langle\prod_{1}^{l+k} \hat{\Sigma}_{\mathrm{v}_{q}}^{x}\right\rangle .
$$


We have,

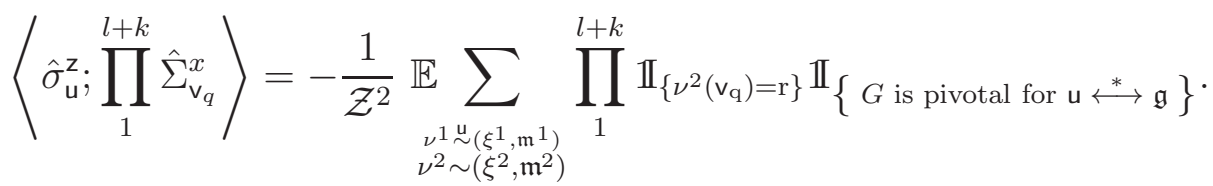

Further correlation inequalities. In the classical case (see e.g. [1,3,4,17]) random current representations of correlations generate a variety of correlation inequalities. In fact, the morphology in the quantum case is even richer and this issue will be systematically addressed in a future paper [11]. Here we shall focus only on such inequalities which are needed for proving our main results.

Partial derivatives with respect to the parameters $(h, \lambda, \rho)$ of the magnetization $M=\left\langle\hat{\sigma}_{0}^{z}\right\rangle$ are related to truncated correlations in the following way: Fix the origin 0 of $\mathbb{T}_{N}$ and let $0 \in \mathfrak{S}$ be the point with the space time coordinates $0=(0,0)$. In view of (space and time) translation invariance it is of course inessential how we fix 0. Then,

$$
\begin{aligned}
& \frac{\partial M}{\partial h}=\sum_{i \in \mathbb{T}_{N}} \int_{0}^{\beta}\left\langle\hat{\sigma}_{0}^{z} ; \hat{\sigma}_{(i, t)}^{\mathrm{z}}\right\rangle \mathrm{d} t, \quad \frac{\partial M}{\partial \rho}=\sum_{(i, j): J_{i-j}>0} \frac{J_{i j}}{2} \int_{0}^{\beta}\left\langle\hat{\sigma}_{0}^{\mathrm{z}} ; \hat{\sigma}_{(i, t)}^{\mathrm{z}} \hat{\sigma}_{(j, t)}^{\mathrm{z}}\right\rangle \mathrm{d} t, \\
& \text { and } \frac{\partial M}{\partial \lambda}=\sum_{i \in \mathbb{T}_{N}} \int_{0}^{\beta}\left\langle\hat{\sigma}_{0}^{\mathrm{z} ;} \hat{\Sigma}_{(i, t)}^{x}\right\rangle \mathrm{d} t .
\end{aligned}
$$

Random current representations for the $\mathbf{z}$ and cross-correlations were already given above. Let us therefore turn to $\left\langle\hat{\sigma}_{0}^{z} ; \hat{\sigma}_{(i, t)}^{z} \hat{\sigma}_{(j, t)}^{z}\right\rangle$ terms. In order to facilitate the notation set $\mathbf{w}=(i, t)$ and $\mathbf{z}=(j, t)$. The random current representation of

$$
\left\langle\hat{\sigma}_{0}^{\mathrm{z}} \hat{\sigma}_{\mathrm{w}}^{\mathrm{z}} \hat{\sigma}_{\mathrm{z}}^{\mathrm{z}}\right\rangle=\left\langle\hat{\sigma}_{0}^{\mathrm{z}} \hat{\sigma}_{\mathrm{w}}^{\mathrm{z}} \hat{\sigma}_{\mathrm{z}}^{\mathrm{z}}\right\rangle \frac{\mathcal{Z}}{\mathcal{Z}}=\frac{1}{\mathcal{Z}^{2}} \mathbb{E} \sum_{\substack{\nu^{1}\{0, \mathrm{w}, \mathrm{z}\} \\ \nu^{2} \sim\left(\xi^{1}, \mathfrak{m}^{1}\right)}} 1,
$$

is straightforward. Consider now,

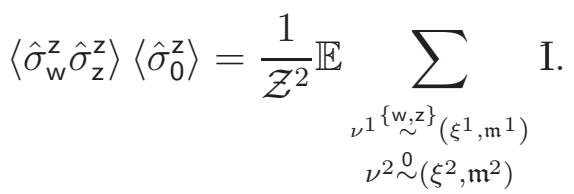

Each pair of triples $\left\{\left(\nu^{1}, \xi^{1}, \mathfrak{m}^{1}\right),\left(\nu^{2}, \xi^{2}, \mathfrak{m}^{2}\right)\right\}$ which contributes to the latter integral contains an unblocked path from 0 to $\mathfrak{g}$. Performing our Basic Transformation along the minimal such path, we infer,

$$
\left\langle\hat{\sigma}_{\mathrm{w}}^{\mathrm{z}} \hat{\sigma}_{\mathrm{z}}^{\mathrm{z}}\right\rangle\left\langle\hat{\sigma}_{0}^{\mathrm{z}}\right\rangle=\frac{1}{\mathcal{Z}^{2}} \mathbb{E} \sum_{\substack { \nu^{1} \\
\begin{subarray}{c}{\{0, \mathrm{w}, \mathrm{z}\} \\
\nu^{2} \sim\left(\xi^{1}, \mathfrak{m}^{2}\right){ \nu ^ { 1 } \\
\begin{subarray} { c } { \{ 0 , \mathrm { w } , \mathrm { z } \} \\
\nu ^ { 2 } \sim ( \xi ^ { 1 } , \mathfrak { m } ^ { 2 } ) } }\end{subarray}} \mathbb{1}_{\{0 \stackrel{*}{\longleftrightarrow} \mathfrak{g}\}}
$$


Consequently,

$$
\left\langle\hat{\sigma}_{0}^{z} ; \hat{\sigma}_{\mathrm{w}}^{\mathrm{z}} \hat{\sigma}_{\mathrm{z}}^{\mathrm{z}}\right\rangle=\frac{1}{\mathcal{Z}^{2}} \mathbb{E} \sum_{\substack{\nu^{1}\{0, \mathfrak{w}, \mathrm{z}\} \\ \nu^{2} \sim\left(\xi^{1}, \mathfrak{m}^{1}\right)}} \mathbb{I}_{\left\{0 \aleph^{*} \rightarrow \mathfrak{g}\right\}} .
$$

In particular, $\partial M / \partial \rho \geq 0$.

One can readily generalize the latter conclusion to a system with inhomogeneous flip rates in the following fashion: Let $\rho_{\mathrm{e}}: \mathbb{S}_{\beta} \mapsto \mathbb{R}_{+}$; e $\in \mathcal{E}^{0}$ be a collection of non-negative (and, say, piece-wise smooth) functions. Let us view the $\rho_{\mathrm{e}}$ 's as timeinhomogeneous rates of arrivals of (ground) flips corresponding to the endpoints of e. In this way, we may introduce an analog of (2.2), defining z-expectation values

$$
M_{\mathrm{u}}(\rho(\cdot))=M_{\mathrm{u}}(h, \rho(\cdot), \lambda) ; \quad \mathrm{u} \in \mathfrak{S},
$$

via the right hand side of (2.2) but using the inhomogeneous arrival rates $\left(\rho_{e}(t)\right)_{e \in \mathcal{E}^{0}}$.

Then, for every $\mathrm{u} \in \mathfrak{S}$, the functional $M_{\mathrm{u}}(\cdot)$ is non-decreasing in $\rho$, that is

$$
\forall \text { e } \rho_{\mathrm{e}} \leq \rho_{\mathrm{e}}^{\prime} \mathrm{t}-\text { a.e. } \quad \Longrightarrow \quad \forall \mathrm{u} M_{\mathrm{u}}(\rho) \leq M_{\mathrm{u}}\left(\rho^{\prime}\right)
$$

It is worth noting that this may be seen as a special case of Griffith's second inequality [13].

Obviously, we may use the random current representation to introduce timeinhomogeneous versions of all correlations we have already encountered in this paper. With that in mind, the following combination of (3.16) with (3.13) will be useful in the sequel: Let $\mathfrak{A}=\mathfrak{I}_{1} \cup \cdots \cup \mathfrak{I}_{n}$ be a union of disjoint ground segments of the form either for some $i ; \mathfrak{I}_{l}=\mathbb{S}_{\beta}^{i}$, or $\mathfrak{I}_{l}=\left(\mathrm{w}_{l}, \mathrm{z}_{l}\right)$, where both $\mathrm{w}_{l}$ and $\mathrm{z}_{l}$ lie on some circle $\mathbb{S}_{\beta}^{i}$ (and are time ordered to avoid notational ambiguities). Define $\mathfrak{A}^{c}=\mathfrak{S} \backslash \mathfrak{A}$. Finally define the reduced arrival rates $\rho^{\mathfrak{A}}$,

$$
\rho_{\mathrm{e}}^{\mathfrak{A}}(t)= \begin{cases}\rho, & \text { if the corresponding flip is either between two points in } \mathfrak{A} \\ & \text { or between two points in } \mathfrak{A}^{c} \\ 0, & \text { otherwise. }\end{cases}
$$

In other words we suppress arrivals of flips between $\mathfrak{A}$ and $\mathfrak{A}^{c}$. Let $u \in \mathfrak{A}^{c}$ and let $\mathrm{v}_{1}, \ldots, \mathrm{v}_{l}, \mathrm{u}, \mathrm{v}_{l+1}, \ldots, \mathrm{v}_{2 n}$ be the time ordering of the set $\left\{\mathrm{w}_{1}, \ldots, \mathrm{z}_{n}, \mathrm{u}\right\}$. Then, exactly as in (3.13),

$$
\left\langle\prod_{1}^{l} \hat{\Sigma}_{\mathbf{v}_{q}}^{x} \hat{\sigma}_{\mathrm{u}}^{z} \prod_{l+1}^{2 n} \hat{\Sigma}_{\mathrm{v}_{q}}^{x}\right\rangle\left(\rho^{\mathfrak{A}}\right) \leq\left\langle\hat{\sigma}_{\mathrm{u}}^{\mathbf{z}}\right\rangle\left(\rho^{\mathfrak{A}}\right)\left\langle\prod_{1}^{2 n} \hat{\Sigma}_{\mathrm{v}_{q}}^{x}\right\rangle\left(\rho^{\mathfrak{A}}\right) \leq M(\rho)\left\langle\prod_{1}^{2 n} \hat{\Sigma}_{\mathrm{v}_{q}}^{x}\right\rangle\left(\rho^{\mathfrak{A}}\right),
$$

where the expectations are understood in terms of the corresponding (generalized to time-inhomogeneous rates) random current representations, and the second inequality follows from (3.16).

In view of how the rates $\rho^{\mathfrak{A}}$ were defined, fixing labels at the end-points of $\mathfrak{I}_{1}, \ldots, \mathfrak{I}_{n}$ completely decouples the two regions $\mathfrak{A}$ and $\mathfrak{A}^{c}$. As a result, we obtain the following inequality:

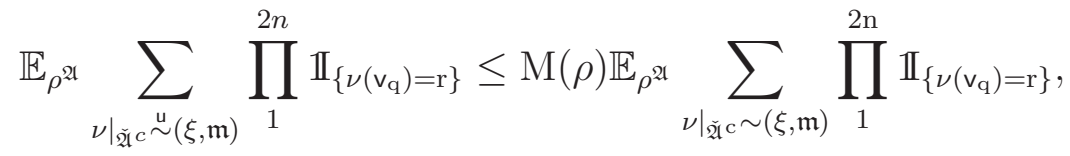


where the expectation above is with respect to $\rho^{\mathfrak{A}}$-arrival rates and the summation is over all reduced labels $\left.\nu\right|_{\mathfrak{A}^{c}}: \mathfrak{A}^{c} \mapsto\{r, l\}$.

\section{Differential inequalities}

The following is an adaptation of the ideas of $[2,3]$ to the quantum case. It is worth noting that the space-time techniques we develop here yield simplified proofs even in the classical case.

A fruitful idea of [3] is to work with three replicas in order to control the above quantities. In our case these will be three independent replicas $\left(\xi^{1}, \mathfrak{m}^{1}\right),\left(\xi^{2}, \mathfrak{m}^{2}\right)$, $\left(\xi^{3}, \mathfrak{m}^{3}\right)$ of Poisson processes of flips and marks and, respectively, three sets of compatible labels $\nu^{1}, \nu^{2}, \nu^{3}$. We shall always indicate in sub-indices which replicas we are talking about, e.g. we shall talk about left $l_{1}$ paths in the first replica or about unblocked $*_{23}$-paths in the replicas 2 and 3 . In the sequel $\mathbb{P}$ is the product measure for all three independent replicas and $\mathbb{E}$ denotes the corresponding expectation.

Let us go back to the representations (2.2) and (2.1),

$$
\left\langle\hat{\sigma}_{0}^{z}\right\rangle=\left\langle\hat{\sigma}_{0}^{z}\right\rangle \frac{\mathcal{Z}^{2}}{\mathcal{Z}^{2}}=\frac{1}{\mathcal{Z}^{3}} \mathbb{E} \sum_{\substack { \nu^{1} \stackrel{0}{\sim}\left(\xi^{1}, \mathfrak{m}^{1}\right) \\
\begin{subarray}{c}{\nu^{2} \sim\left(\xi^{2}, \mathfrak{m}^{2}\right) \\
\nu^{3} \sim\left(\xi^{3}, \mathfrak{m}^{3}\right){ \nu ^ { 1 } \stackrel { 0 } { \sim } ( \xi ^ { 1 } , \mathfrak { m } ^ { 1 } ) \\
\begin{subarray} { c } { \nu ^ { 2 } \sim ( \xi ^ { 2 } , \mathfrak { m } ^ { 2 } ) \\
\nu ^ { 3 } \sim ( \xi ^ { 3 } , \mathfrak { m } ^ { 3 } ) } }\end{subarray}} 1
$$

Let $\mathrm{C}_{23}^{*}(\mathfrak{g})$ be the set of all points $\vee \in \mathfrak{S}$ which are $*_{23}$-connected to $\mathfrak{g}$ and let us denote $\check{C}_{1}^{l}(0, \mathfrak{g})$ as the set of ground $(\mathfrak{S})$ points on the unique ground left path from 0 to $\mathfrak{g}$. We shall distinguish three cases which exhaust all possible contributions to the right hand side of (4.1) and lead to the various terms in (1.3):

(1) $\check{\mathrm{C}}_{1}^{l}(0, \mathfrak{g}) \cap \mathrm{C}_{23}^{*}(\mathfrak{g})=\emptyset$.

(2) $0 \in \mathrm{C}_{23}^{*}(\mathfrak{g})$.

(3) $0 \notin \mathrm{C}_{23}^{*}(\mathfrak{g})$ but $\check{\mathrm{C}}_{1}^{l}(0, \mathfrak{g}) \cap \mathrm{C}_{23}^{*}(\mathfrak{g}) \neq \emptyset$.

Below we consider these cases in turn (see Figure 4 ). During our exposition of CASE 3, we also derive the pair of inequalities (1.4).

CASE 2 If $0 \in \mathrm{C}_{23}^{*}(\mathfrak{g})$ then there exist $*_{23}$-paths from 0 to $\mathfrak{g}$. Hence the notion of the minimal path $\mathcal{P}^{*} \triangleq \mathrm{C}_{23}^{*}(0, \mathfrak{g})$ from 0 to $\mathfrak{g}$ is well defined. Applying the Basic Transformation $\Phi_{\mathcal{P}^{*}}$ on 23-labels, we readily conclude,

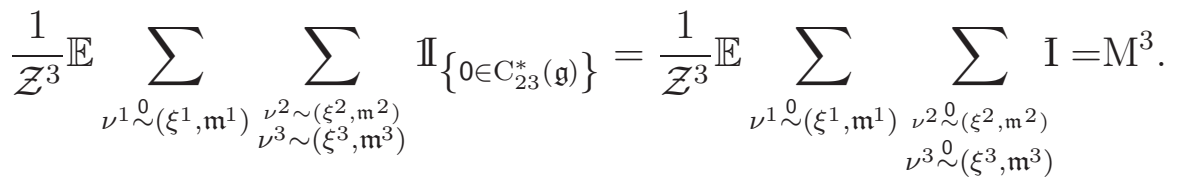

CASE 1 By construction, $\check{\mathrm{C}}_{1}^{l}=\left(\mathfrak{I}_{1}, \ldots, \mathfrak{I}_{n}\right)$. All the intervals in this sequence are ground, and the last interval $\mathfrak{I}_{n}=(\mathrm{w}, \mathrm{v} \triangleq(i, t))$ satisfies $t \in \xi_{i \mathfrak{g}}^{1}$. Let $\widetilde{\xi}^{1}$ be the modified realization of 1-process of flips with the corresponding arrival removed, but the configuration $\left(\nu^{1}, \xi^{1}, \mathfrak{m}^{1}\right)$ otherwise kept intact. Obviously, the relative weight of removing this arrival contributes a factor $h \mathrm{~d} t$, and one can recover the original $\xi^{1}$ by adding a flip from $v$ to the ghost site $\mathfrak{g}$. Formally, fixing realizations of the second and third replicas and fixing compatible values of $\nu^{1}$ and $\nu^{2}$, taking expectations 


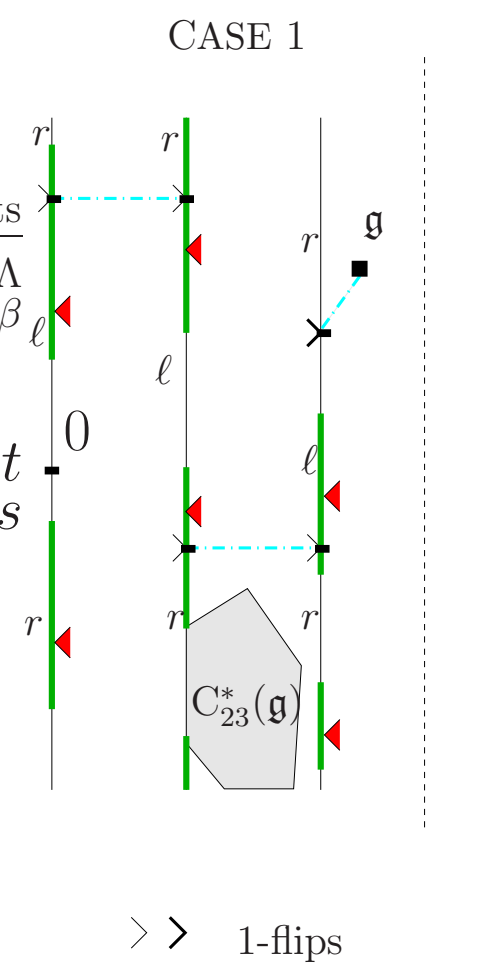

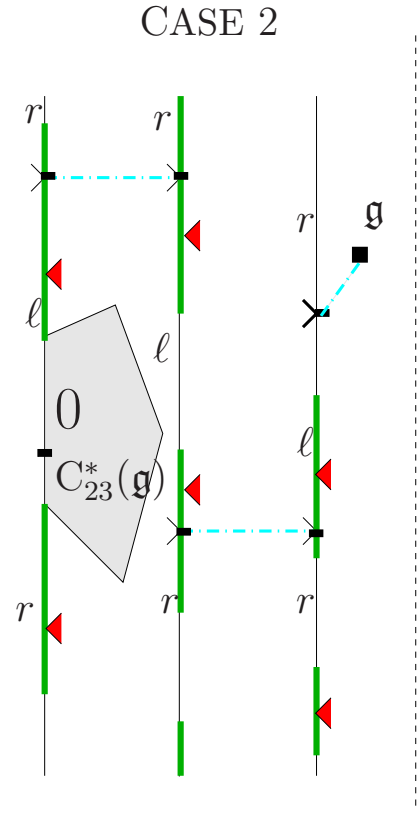

\23-marks
CASE 3

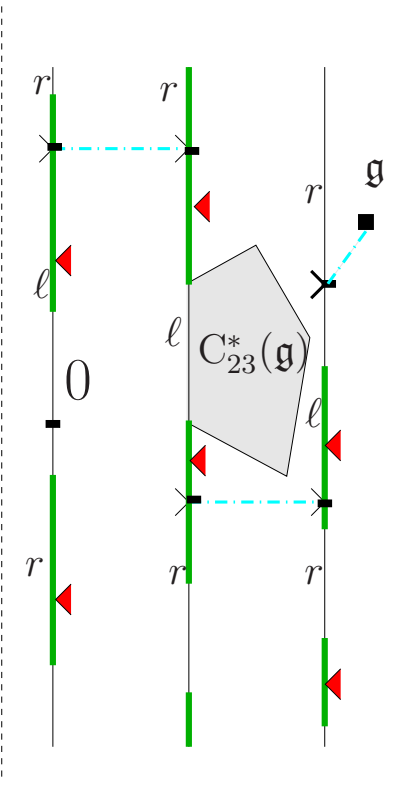

Blocked 23-intervals

Figure 4. The ground left 1-path $\check{C}_{1}^{l}(0, \mathfrak{g})$ contains three intervals. $r$ and $\ell$ are $\nu^{1}$-labels of the first replica. The unblocked 23-cluster $\mathrm{C}_{23}^{*}(\mathfrak{g})$ is depicted schematically.

CASE 1: $\check{C}_{1}^{l}(0, \mathfrak{g})$ is disjoint from $\mathrm{C}_{23}^{*}(\mathfrak{g})$. CASE 2: $0 \in \mathrm{C}_{23}^{*}(\mathfrak{g})$. CASE 3: $0 \notin \mathrm{C}_{23}^{*}(\mathfrak{g})$, but $\check{\mathrm{C}}_{1}^{l}(0, \mathfrak{g}) \cap \mathrm{C}_{23}^{*}(\mathfrak{g}) \neq \emptyset$.

only with respect to $\left(\xi^{1}, \mathfrak{m}^{1}\right)$ and summing only with respect to compatible $\nu^{1}$-labels we obtain

$$
\begin{aligned}
& \mathbb{E}_{1}\left[\sum_{\nu^{1} \stackrel{0}{\sim}\left(\xi^{1}, \mathfrak{m}^{1}\right)} \mathbb{I}_{\left\{\check{\mathrm{C}}_{1}^{l}(0, \mathfrak{g}) \cap C_{23}^{*}(\mathfrak{g})=\varnothing\right\}}\right] \\
& =\sum_{i \in \mathbb{T}_{N}} \int_{0}^{\beta} h \mathrm{~d} t \mathbb{E}_{1}\left[\sum_{\substack{\nu^{1} \sim\left(\xi^{1}, \mathfrak{m}^{1}\right) \\
\left\{\check{C}_{1}^{\mathrm{l}}(0, \mathfrak{g}) \cap C_{23}^{*}(\mathfrak{g})=\varnothing\right\}}} \mathbb{I}_{\left\{(\mathrm{i}, \mathrm{t}) \in \check{\mathrm{C}}_{1}^{1}(0, \mathfrak{g})\right\}} \mid \xi_{\mathrm{i}, \mathfrak{g}}^{1}(\mathrm{t})=1\right]
\end{aligned}
$$


Now ,

$$
\begin{aligned}
& \mathbb{E}_{1}\left[\sum_{\nu^{1} \stackrel{0}{\sim}\left(\xi^{1}, \mathfrak{m}^{1}\right)} \mathbb{I}_{\left\{\check{\mathrm{C}}_{1}^{\mathrm{l}}(0, \mathfrak{g}) \cap \mathrm{C}_{23}^{*}(\mathfrak{g})=\varnothing\right\}} \mathbb{I}_{\left\{(\mathrm{i}, \mathrm{t}) \in \check{C}_{1}^{\mathrm{l}}(0, \mathfrak{g})\right\}} \mid \xi_{\mathrm{i}, \mathfrak{g}}^{1}(\mathrm{t})=1\right] \\
& =\mathbb{E}_{1}\left[\sum_{\nu^{1, v v}\left(\xi^{1}, \mathfrak{m}^{1}\right)} \mathbb{1}_{\left\{\mathrm{C}_{1}^{1}(0, v) \cap C_{23}^{*}(\mathfrak{g})=\varnothing\right\}}\right]
\end{aligned}
$$

with $\mathbf{v}=(i, t)$ on the right-hand side.

Taking into account replicas 2 and 3, let us determine the properties of the resulting triple of configurations from the joint integration on the right-hand side of (4.4). Since $\mathrm{C}_{1}^{l}(0, \mathrm{v}) \cap \mathrm{C}_{23}^{*}(\mathfrak{g})=\varnothing$, there exist $*_{12}$-paths from 0 to $\mathrm{v}$ which are disjoint from $\mathrm{C}_{23}^{*}(\mathfrak{g})$. Let $\mathcal{P}^{*}$ be the minimal such path. Consider the Basic Transformation $\Phi_{\mathcal{P}^{*}}$ on 12-labels. It produces new collection $\left\{\left(\hat{\nu}^{1}, \hat{\xi}^{1}, \hat{\mathfrak{m}}^{1}\right),\left(\nu^{2}, \hat{\xi}^{2}, \hat{\mathfrak{m}}^{2}\right)\right\}$, which satisfies the following set of conditions:

(1) $\mathcal{P}^{*}$ is still the minimal $*_{12}$ path from 0 to $\boldsymbol{v}$ which avoids $\mathrm{C}_{23}^{*}(\mathfrak{g})$. In particular, the transformation is invertible and $0 \stackrel{*^{23}}{\longrightarrow} \mathfrak{g}$

(2) $\hat{\nu}^{1} \sim\left(\hat{\xi}^{1}, \hat{\mathfrak{m}}^{1}\right)$ and $\hat{\nu}^{2} \stackrel{0, v}{\sim}\left(\hat{\xi}^{2}, \hat{\mathfrak{m}}^{2}\right)$. In particular, $0 \stackrel{*_{23}}{\longleftrightarrow} \mathrm{v}$.

Comparing with (3.5) (applied to 2 and 3 labels) and with the first of (3.14), we conclude

$$
\frac{1}{\mathcal{Z}^{3}} \mathbb{E} \sum_{\substack { \nu^{1} \sim\left(\xi^{1}, \mathfrak{m}^{1}\right) \\
\begin{subarray}{c}{\nu^{2} \sim\left(\xi^{2}, \mathfrak{m}^{2}\right) \\
\nu^{3} \sim\left(\xi^{3}, \mathfrak{m}^{3}\right){ \nu ^ { 1 } \sim ( \xi ^ { 1 } , \mathfrak { m } ^ { 1 } ) \\
\begin{subarray} { c } { \nu ^ { 2 } \sim ( \xi ^ { 2 } , \mathfrak { m } ^ { 2 } ) \\
\nu ^ { 3 } \sim ( \xi ^ { 3 } , \mathfrak { m } ^ { 3 } ) } }\end{subarray}} \mathbb{I}_{\left\{\check{\mathrm{C}}_{1}^{1}(0, \mathfrak{g}) \cap \mathrm{C}_{23}^{*}(\mathfrak{g})=\emptyset\right\}} \leq \sum_{\mathrm{i}} \int_{0}^{\beta} \operatorname{hdt}\left\langle\hat{\sigma}_{0}^{\mathrm{z}} ; \hat{\sigma}_{(\mathrm{i}, \mathrm{t})}^{\mathrm{z}}\right\rangle=\mathrm{h} \frac{\partial \mathrm{M}}{\partial \mathrm{h}} .
$$

CASE 3 This is the most difficult case. In fact it contains two sub-cases, which we proceed to describe:

The left ground path from 0 to $\mathfrak{g}$, denoted by $\check{C}_{1}^{l}(0, \mathfrak{g})$, is a ground path which may be naturally written as an ordered collection of ground intervals, $\check{C}_{1}^{l}(0, \mathfrak{g})=\cup_{1}^{n} \mathfrak{I}_{l}$ : Each interval $\mathfrak{I}_{l} \triangleq\left[\mathrm{z}_{l}, \mathrm{w}_{l}\right]$ is also naturally oriented with respect to the direction of the path towards $\mathfrak{g}$. Therefore, in the case under consideration we can speak of the first interval $\mathfrak{I}_{l^{*}}$ where $\check{C}_{1}^{l}(0, \mathfrak{g})$ hits $\mathrm{C}_{23}^{*}(\mathfrak{g})$ and, furthermore about the first hitting point $\mathbf{u}^{*} \in \mathfrak{I}_{l^{*}}$.

CASE 3(a) Pivotal Marks: In this sub-case $\mathbf{z}_{l^{*}} \stackrel{*_{23}}{\longrightarrow} \mathfrak{g}$ or, equivalently, $\mathbf{z}_{l^{*}} \neq \mathbf{u}^{*}$. Since $\mathbf{u}^{*}$ is in the boundary of $\mathrm{C}_{23}^{*}(\mathfrak{g})$, there is a necessarily a 23-mark at $\mathbf{u}^{*}$. Also, both the 2 and 3 labels are $r$ at $\mathbf{u}^{*}$. By construction, (if we understand the interval $\left(\mathbf{z}_{l^{*}}, \mathbf{u}^{*}\right)$ as being topologically open $)$

$$
\check{\mathrm{C}}_{1}^{l}\left(0, \mathrm{u}^{*}\right) \cap \mathrm{C}_{23}^{*}(\mathfrak{g})=\emptyset .
$$

Hence there exist $*_{12}$-paths from 0 to $\mathrm{u}^{*}$ which avoid $\mathrm{C}_{23}^{*}(\mathfrak{g})$. Let $\mathcal{P}_{12}^{*}\left(0, \mathrm{u}^{*}\right)$ be the minimal such path. Let also $\mathcal{P}_{23}^{*}\left(\mathbf{u}^{*}, \mathfrak{g}\right)$ be the minimal $*_{23}$-path from $\mathbf{u}^{*}$ to $\mathfrak{g}$. These 
paths are disjoint. Let us make the following double transformation on all three collections of replicas and compatible labels:

(1) Remove the 23-mark at $\mathrm{u}^{*}$. This yields the weight $2 \lambda \mathrm{d} t$.

(2) Perform the Basic Transformation $\Phi_{\mathcal{P}_{12}^{*}\left(0, u^{*}\right)}$ on 12-labels.

(3) Perform the Basic Transformation $\Phi_{\mathcal{P}_{23}^{*}\left(\mathbf{u}^{*}, \mathfrak{g}\right)}$ on 23-labels.

Since the Basic Transformations are on disjoint paths the latter two operations are well defined, commute and moreover do not change the minimal character of $\mathcal{P}_{12}^{*}\left(0, \mathbf{u}^{*}\right)$ and $\mathcal{P}_{23}^{*}\left(\mathbf{u}^{*}, \mathfrak{g}\right)$. In other words, they are invertible. The resulting set of triples

$\left\{\left(\hat{\nu}^{1}, \hat{\xi}^{1}, \hat{\mathfrak{m}}^{1}\right),\left(\hat{\nu}^{2}, \hat{\xi}^{2}, \hat{\mathfrak{m}}^{2}\right),\left(\hat{\nu}^{3}, \hat{\xi}^{3}, \hat{\mathfrak{m}}^{3}\right)\right\}$ satisfies the following conditions (see Figure 5):

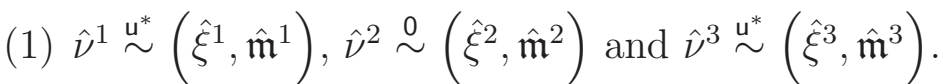

(2) $\hat{\nu}^{2}\left(u^{*}\right)=l$.

(3) $\mathrm{u}^{*}$ is pivotal for $\left\{0 \stackrel{*_{23}}{\longleftrightarrow} \mathfrak{g}\right\}$.

Note that (2) is a consequence of (1) and (3).

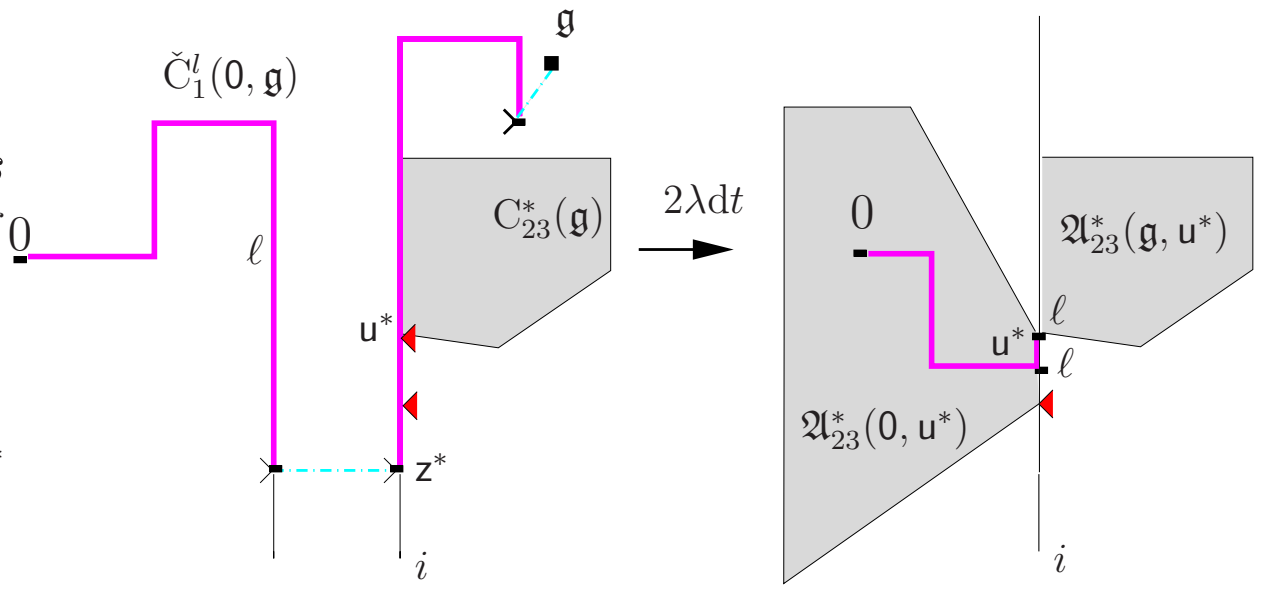

$>$ 1-flips $\triangleleft$ 23-marks $\mid$ (Left) Left ground 1-path $\check{C}_{1}^{l}(0, \mathfrak{g})$

| (Right) Minimal 12-path $\mathcal{P}_{12}^{*}\left(0, \mathrm{u}^{*}\right)$

Figure 5. Double transformation in the CASE 3 (a): The 23-mark at $\mathrm{u}^{*}$ is removed at the cost $2 \lambda \mathrm{d} t$. The point $\mathrm{u}^{*}$ is pivotal for the $\left\{0 \stackrel{*_{23}}{\longleftrightarrow} \mathfrak{g}\right\}$-connection in the modified configuration. $\mathfrak{A}_{23}^{*}\left(0, \mathbf{u}^{*}\right)$ is the set of all the points $\mathrm{u} \in \mathfrak{S}$ which can be reached from 0 via unblocked 23-paths avoiding $\mathbf{u}^{*} . \mathfrak{A}_{23}^{*}\left(\mathfrak{g}, \mathrm{u}^{*}\right)$ is the set of all the points $\mathrm{u} \in \mathfrak{S}$ which can be reached from $\mathfrak{g}$ via unblocked 23-paths avoiding $\mathbf{u}^{*}$. 
We claim that,

$$
\begin{aligned}
& \mathbb{E} \sum_{\nu^{2} \sim \underset{\sim}{\sim}\left(\xi^{2}, \mathfrak{m}^{2}\right)} \mathbb{I}_{\left\{\mathrm{u}^{*} \text { is pivotal for } 0 \stackrel{*_{23}}{\longleftrightarrow} \mathfrak{g}\right\}} \mathbb{I}_{\left\{\nu^{2}\left(\mathbf{u}^{*}\right)=l\right\}}
\end{aligned}
$$

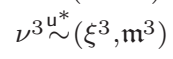

$$
\begin{aligned}
& \leq M \mathbb{E} \sum_{\substack{\nu^{2} \sim\left(\xi^{2}, \mathfrak{m}^{2}\right) \\
\nu^{3} \sim\left(\xi^{3}, \mathfrak{m}^{3}\right)}} \mathbb{I}_{\left\{\mathrm{u}^{*} \text { is pivotal for } 0 \stackrel{*_{23}}{\longleftrightarrow} \mathfrak{g}\right\}} \mathbb{I}_{\left\{\nu^{3}\left(\mathrm{u}^{*}\right)=\mathrm{r}\right\}} .
\end{aligned}
$$

Assuming (4.7) for the moment, a comparison with (3.12) and with the third of (3.14) reveals that the total contribution to $M$ which comes from the CASE 3(a) is bounded above by

$$
M^{2} \frac{1}{\mathcal{Z}^{2}} \mathbb{E} \sum_{i} \int_{0}^{\beta} 2 \lambda \mathrm{d} t \sum_{\substack{\nu^{2} \stackrel{0}{\sim}\left(\xi^{2}, \mathfrak{m}^{2}\right) \\ \nu^{3} \sim\left(\xi^{3}, \mathfrak{m}^{3}\right)}} \mathbb{I}_{\left\{(i, t) \text { is pivotal for } 0 \stackrel{*_{23}}{\longleftrightarrow} \mathfrak{g}\right\}} \mathbb{I}_{\left\{\nu^{3}\left(\mathbf{u}^{*}\right)=\mathrm{r}\right\}}=-2 \lambda \mathrm{M}^{2} \frac{\partial \mathrm{M}}{\partial \lambda}
$$

To check (4.7) let $\mathfrak{A}_{23}^{*}\left(0, \mathrm{u}^{*}\right)$ be the set of all the points $\mathrm{u} \in \mathfrak{S}$ which can be reached from 0 via unblocked 23-paths avoiding $\mathbf{u}^{*}$. Evidently, $\mathfrak{A}_{23}^{*}\left(0, \mathbf{u}^{*}\right)$ can be written as a union of intervals $\mathfrak{A}_{23}^{*}\left(0, \mathbf{u}^{*}\right)=\cup \mathcal{R}_{j}$, which satisfy the following set of properties:

(1) Each interval $\mathcal{R}_{j} \triangleq\left(\mathrm{p}_{j}, \mathrm{q}_{j}\right)$ (which formally speaking union of successive ground intervals on some $\mathbb{S}_{\beta}^{i}$ ) bears 23-marks at its endpoints $\mathrm{p}_{j}$ and $\mathbf{q}_{j}$, except, of course, for the interval which contains $u^{*}$ as one of its endpoints recall that the 23-mark at $\mathbf{u}^{*}$ was removed. Moreover, both labels $\nu^{2}$ and $\nu^{3}$ equal to $r$ at such end-points.

(2) Let $\mathcal{R}_{j^{*}}=\left(\mathrm{p}_{j^{*}}, \mathrm{u}^{*}\right)$ be the remaining interval which contains $\mathrm{u}^{*}$ as one of its

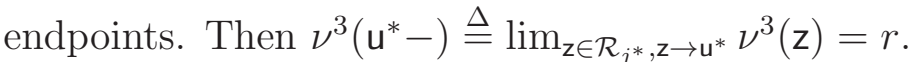

(3) There are no arrivals of 23-flips between points in $\mathfrak{A}_{23}^{*}\left(0, \mathbf{u}^{*}\right)$ and points in $\mathfrak{S} \backslash \mathfrak{A}_{23}^{*}\left(0, \mathbf{u}^{*}\right)$.

The inequality (4.7) is then proved as follows: Conditioning on $\mathfrak{A}_{23}^{*}\left(0, \mathrm{u}^{*}\right)$ with realizations of all the processes and values of both 2 and 3 labels on it, we integrate with respect to marks on $\mathfrak{S} \backslash \mathfrak{A}_{23}^{*}\left(0, \mathbf{u}^{*}\right)$, flips on $\mathfrak{S} \backslash \mathfrak{A}_{23}^{*}\left(0, \mathbf{u}^{*}\right) \cup \mathfrak{g}$ and compatible 2 and 3 labels. The constrained integration clearly decouples the two configurations on $\mathfrak{S} \backslash \mathfrak{A}_{23}^{*}\left(0, \mathbf{u}^{*}\right) \cup \mathfrak{g}$ and so we can integrate the restricted 2 and 3 quantities independently. We arrive at a situation where (3.18) applies (for the restriction of $\left.\nu^{3}\right)$. More precisely, what we use is actually a limiting case of (3.18), with the $z$ component of spin in the expectation occurring at the point $\mathrm{u}^{*}$ on the boundary of $\mathfrak{S} \backslash \mathfrak{A}_{23}^{*}\left(0, \mathbf{u}^{*}\right) \triangleq \mathfrak{A}_{23}^{*}\left(0, \mathbf{u}^{*}\right)^{c}$. Putting things together concludes Step 3(a).

Before proceeding to CASE 3(b), let us prove the first of (1.4) by techniques similar to those of the previous paragraph. With the same notation as in Step 3(a), recall that $\mathfrak{A}_{23}^{*}\left(0, \mathrm{u}^{*}\right) \cap \mathrm{C}_{23}^{*}(\mathfrak{g})=\emptyset$. Consequently, by (3.18) (or, more precisely by the limiting case of the latter, again applied to the restriction of $\nu^{2}$ to $\mathfrak{A}_{23}^{*}\left(0, \mathbf{u}^{*}\right)^{c}$ at 
the point $\mathbf{u}^{*}$ on the boundary of $\left.\mathfrak{A}_{23}^{*}\left(0, \mathbf{u}^{*}\right)^{c}\right)$,

$\mathbb{E} \sum_{\substack{\nu^{2} \stackrel{0}{\sim}\left(\xi^{2}, \mathfrak{m}^{2}\right) \\ \nu^{3} \sim\left(\xi^{3}, \mathfrak{m}^{3}\right)}} \mathbb{I}_{\left\{\mathrm{u}^{*} \text { is pivotal for } 0 \stackrel{*_{23}}{\longrightarrow} \mathfrak{g}\right\}} \mathbb{I}_{\left\{\nu^{3}\left(\mathrm{u}^{*}\right)=\mathrm{r}\right\}} \leq \mathrm{ME} \sum_{\substack{\nu^{2}, \mathrm{u}^{*}\left(\xi^{2}, \mathfrak{m}^{2}\right) \\ \nu^{3} \sim\left(\xi^{3}, \mathfrak{m}^{3}\right)}} \mathbb{I}_{\left\{\mathfrak{A}_{23}^{*}\left(0, \mathrm{u}^{*}\right) \cap \mathrm{C}_{23}^{*}(\mathfrak{g})=\emptyset\right\}} \mathbb{I}_{\left\{\nu^{3}\left(\mathrm{u}^{*}\right)=\mathrm{r}\right\}}$

Now

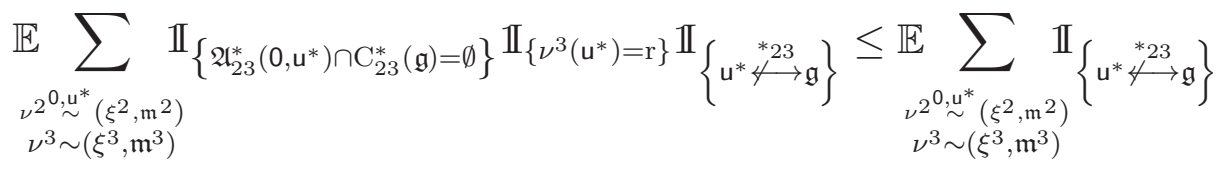

and the right-hand side is $\mathcal{Z}^{2}\left\langle\hat{\sigma}_{0}^{z} ; \hat{\sigma}_{\mathrm{u}^{*}}^{\mathrm{z}}\right\rangle$ (see (3.5)$)$. On the other hand

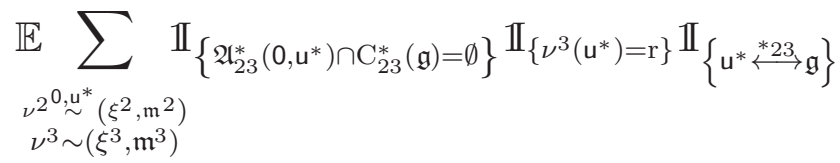

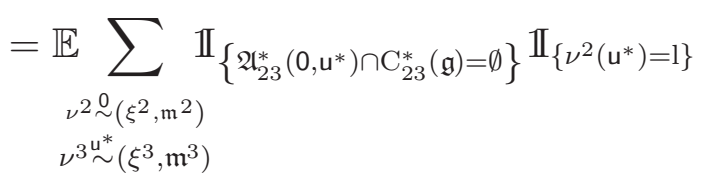

as can be seen by performing our Basic Transformation on the minimal $*_{23}$ path from $\mathrm{u}^{*}$ to $\mathfrak{g}$ (which would necessarily lie in $\left.\mathfrak{A}_{23}^{*}\left(0, \mathbf{u}^{*}\right)^{c}\right)$. Since the constraints appearing on the right-hand side imply that $u^{*}$ is pivotal, we may use (4.7) and bound the right hand-side in (4.9) by $-M^{2} \mathcal{Z}^{2}\left\langle\hat{\sigma}_{0}^{z} ; \hat{\Sigma}_{\mathbf{u}^{*}}^{x}\right\rangle$. The inequality (1.4) then follows easily.

CASE 3(b) Pivotal Flips: Assume now that $\mathrm{z}_{l^{*}} \stackrel{*_{23}}{\longleftrightarrow} \mathfrak{g}$ or, equivalently, that $\mathrm{z}_{l^{*}} \in \mathrm{C}_{23}^{*}(\mathfrak{g})$. In order to simplify notation set $\mathrm{z}^{*}=\mathrm{z}_{l^{*}}$ and $\mathrm{w}^{*}=\mathrm{w}_{l^{*}-1}$. Under the above assumption $\mathrm{C}_{23}^{*}(\mathfrak{g})$ is disjoint from the left path $\mathrm{C}_{1}^{l}\left(0, \mathrm{w}^{*}\right)$. Hence there exist $*_{12}$-paths from 0 to $\mathrm{w}^{*}$ which avoid $\mathrm{C}_{23}^{*}(\mathfrak{g})$. Let $\mathcal{P}_{12}^{*}\left(0, \mathrm{w}^{*}\right)$ be the minimal such path. Let also $\mathcal{P}_{23}^{*}\left(\mathbf{z}^{*}, \mathfrak{g}\right)$ be the minimal $*_{23}$-path from $\mathbf{z}^{*}$ to $\mathfrak{g}$. These paths are disjoint. Let us make now the following transformation on all three replicas and labels:

(1) Remove the arrival of $\xi^{1}$ between $w^{*}$ and $z^{*}$, yielding the weight $\rho J_{i, j} \mathrm{~d} t$.

(2) Perform the Basic Transformation $\Phi_{\mathcal{P}_{12}^{*}\left(0, w^{*}\right)}$ on 12-labels.

(3) Perform the Basic Transformation $\Phi_{\mathcal{P}_{23}^{*}\left(z^{*}, \mathfrak{g}\right)}$ on 23-labels.

Again, since the Basic Transformations are on disjoint paths they are well defined and do not change the minimal character of $\mathcal{P}_{12}^{*}\left(0, w^{*}\right)$ and $\mathcal{P}_{23}^{*}\left(\mathbf{z}^{*}, \mathfrak{g}\right)$. Thus, they are invertible and the resulting collection of configurations

$\left\{\left(\hat{\nu}^{1}, \hat{\xi}^{1}, \hat{\mathfrak{m}}^{1}\right),\left(\hat{\nu}^{2}, \hat{\xi}^{2}, \hat{\mathfrak{m}}^{2}\right),\left(\hat{\nu}^{3}, \hat{\xi}^{3}, \hat{\mathfrak{m}}^{3}\right)\right\}$ satisfy the following set of conditions (see Figure 6):

(1) $\hat{\nu}^{1} \mathrm{z}^{*}\left(\hat{\xi}^{1}, \hat{\mathfrak{m}}^{1}\right), \hat{\nu}^{2} \stackrel{\left\{0, \mathrm{w}^{*}, \mathrm{z}^{*}\right\}}{\sim}\left(\hat{\xi}^{2}, \hat{\mathfrak{m}}^{2}\right)$ and $\hat{\nu}^{3} \stackrel{\mathrm{z}^{*}}{\sim}\left(\hat{\xi}^{3}, \hat{\mathfrak{m}}^{3}\right)$.

(2) $\mathrm{C}_{23}^{*}\left(0, \mathrm{w}^{*}\right)$ and $\mathrm{C}_{23}^{*}(\mathfrak{g})$ are disjoint. 


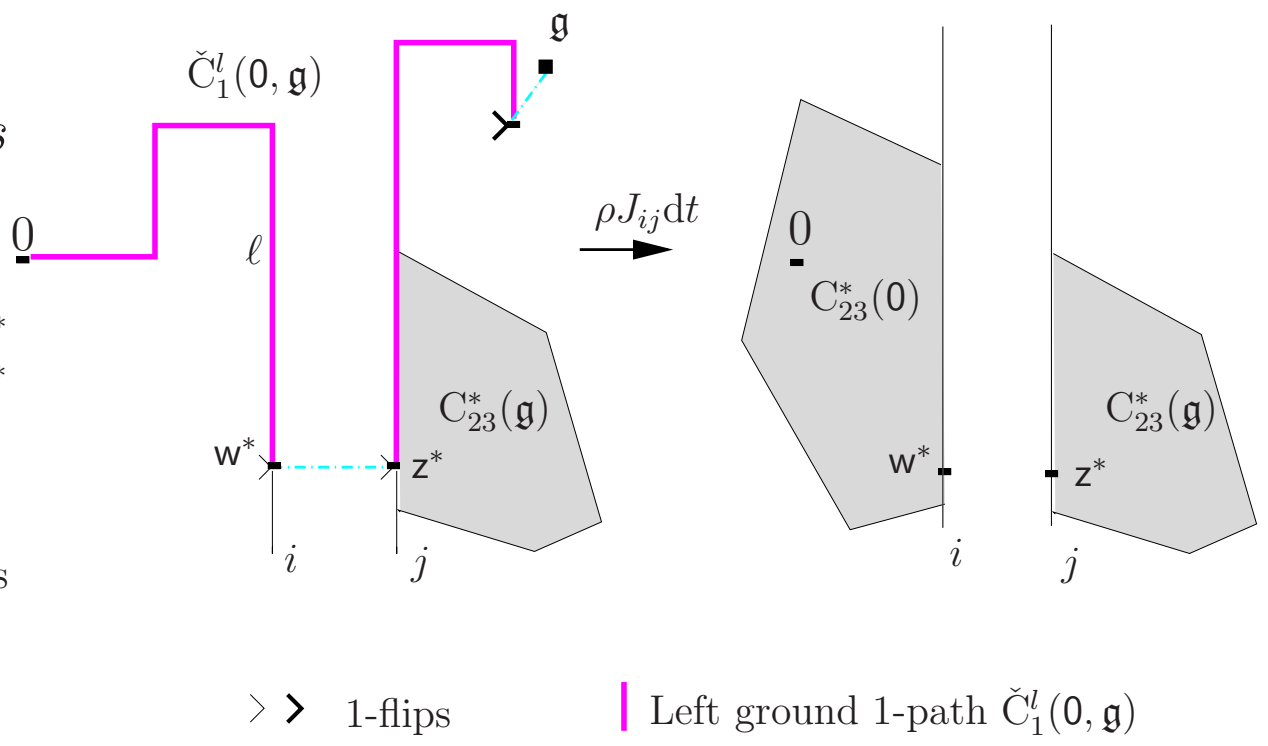

Figure 6. Double transformation in the CASE 3 (b): The 1-flip between $\mathrm{w}^{*}$ at $\mathrm{z}^{*}$ is removed at the cost $\rho J_{i j} \mathrm{~d} t$. In the modified configuration $\mathrm{w}^{*} \in \mathrm{C}_{23}^{*}(0)$ and the clusters $\mathrm{C}_{23}^{*}(0)$ and $\mathrm{C}_{23}^{*}(\mathfrak{g})$ are disjoint.

Therefore, the contribution to $M$ which comes from the CASE 3(b) is bounded by

$$
M \frac{1}{\mathcal{Z}^{2}} \mathbb{E} \sum_{i, j} \int_{0}^{\beta} \rho J_{i j} \mathrm{~d} t \sum_{\substack { \nu^{2}\{0,(i, t),(j, t)\} \\
\begin{subarray}{c}{\nu^{3}(j, t) \\
\nu^{2}\left(\xi^{2}, \mathfrak{m}^{2}\right){ \nu ^ { 2 } \{ 0 , ( i , t ) , ( j , t ) \} \\
\begin{subarray} { c } { \nu ^ { 3 } ( j , t ) \\
\nu ^ { 2 } ( \xi ^ { 2 } , \mathfrak { m } ^ { 2 } ) } }\end{subarray}} \mathbb{I}_{\left\{0 \stackrel{\left.\mathfrak{m}^{3}\right)}{\longleftrightarrow}(\mathrm{i}, \mathrm{t})\right\}} \mathbb{I}_{\left\{\mathrm{C}_{23}^{*}(0,(\mathrm{i}, \mathrm{t})) \cap \mathrm{C}_{23}^{*}(\mathfrak{g})=\emptyset\right\}}
$$

We claim that the latter expression is bounded above by

$$
M^{2} \frac{1}{\mathcal{Z}^{2}} \mathbb{E} \sum_{i, j} \int_{0}^{\beta} \rho J_{i j} \mathrm{~d} t \sum_{\substack{\nu^{2}\{0,(i, t),(j, t)\} \\ \nu^{3} \sim\left(\xi^{3}, \xi^{3}\right)}} \mathbb{I}_{\left\{0 \stackrel{\left.\mathfrak{m}^{2}\right)}{ }\right.} \underset{\left.{ }^{* 23}(\mathrm{i}, \mathrm{t})\right\}}{ } \mathbb{I}_{\left\{\mathrm{C}_{23}^{*}(0,(\mathrm{i}, \mathrm{t})) \cap \mathrm{C}_{23}^{*}(\mathfrak{g})=\emptyset\right\}} .
$$

The proof is the same as that of (4.7) and is omitted here.

The expression in (4.11) is exactly $M^{2} \rho \partial M / \partial \rho$. Indeed, just compare it with (3.15): If we define $\mathbf{w}=(i, t)$ and $\mathbf{z}=(j, t)$, then $0 \stackrel{*^{23}}{\longleftrightarrow} \mathfrak{g}$ precisely means that either $0 \stackrel{*_{23}}{\longleftrightarrow} \mathrm{w}, \mathrm{z} \stackrel{*_{23}}{\longleftrightarrow} \mathfrak{g}$ and $\mathrm{C}_{23}^{*}(0, \mathrm{w}) \cap \mathrm{C}_{23}^{*}(\mathrm{z}, \mathfrak{g})=\emptyset$ or, the other way around, $0 \stackrel{*_{23}}{\longleftrightarrow} \mathrm{z}, \mathrm{w} \stackrel{*_{23}}{\longleftrightarrow} \mathfrak{g}$ and $\mathrm{C}_{23}^{*}(0, \mathrm{z}) \cap \mathrm{C}_{23}^{*}(\mathrm{w}, \mathfrak{g})=\emptyset$.

The second inequality of (1.4) is also an immediate consequence. From a (by now) standard application of the Basic Transformation,

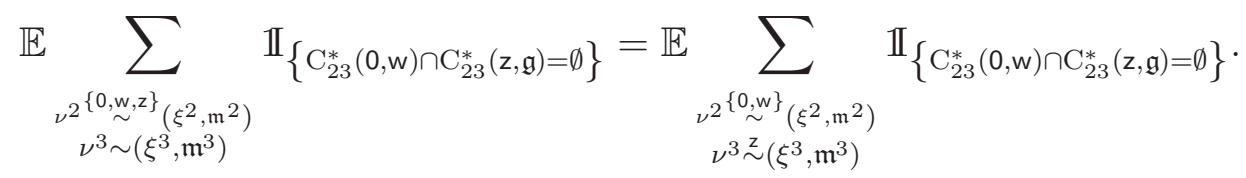


By (3.18) and in view of the representation (3.5),

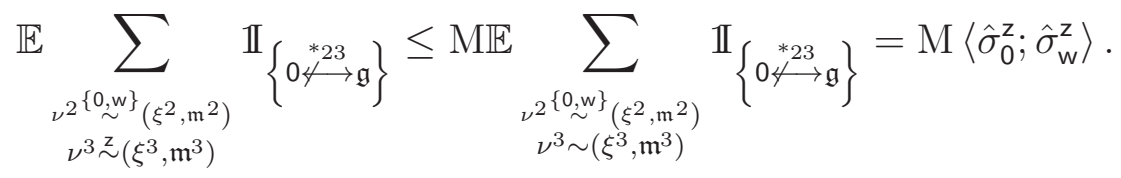

The analogous statement holds if the roles of $z$ and $w$ are interchanged. The conclusion follows by collecting terms.

\section{Proof of Theorem A: Exponential Decay}

In the sequel we shall continue to use $\mathbb{P}$ and, respectively, $\mathbb{E}$ for the product probability for two independent replicas $\left(\xi^{1}, \mathfrak{m}^{1}\right)$ and $\left(\xi^{2}, \mathfrak{m}^{2}\right)$. As before $\mathfrak{n}=\mathfrak{m}^{1} \cup \mathfrak{m}^{2}$ and $\eta=\xi^{1} \cup \xi^{2}$.

The proof is given in three subsections, corresponding to each of the three truncated correlations. The proof for z-correlations is given in some detail and, as the proofs of the second two inequalities only require small modifications of this result, we will be more brief in proving the last two statements.

Proof of Theorem $A$ for z-correlations. Let $i, j \in \mathbb{T}_{N}, s, t \in \mathbb{S}_{\beta}$ be fixed and let $\mathrm{u}=(i, t), \mathbf{v}=(j, s)$. We shall prove the following generalization of the first of (1.2)

Lemma 5.1. There exist $c_{1}=c_{1}(h, \lambda, \rho)>0$ and $c_{2}=c_{2}(h, \lambda, \rho)<\infty$ such that,

$$
\left\langle\hat{\sigma}_{\mathrm{u}}^{\mathrm{z}} ; \hat{\sigma}_{\mathrm{v}}^{\mathrm{z}}\right\rangle \leq c_{2} \mathrm{e}^{-c_{1} \mathrm{~d}(u, v)}
$$

where $\mathrm{d}(\mathrm{u}, \mathrm{v}) \triangleq|j-i|+|t-s|$. The above inequality is uniform in $N, \beta, \mathrm{u}$ and $\mathrm{v}$.

Proof. The starting point for our analysis is the formula (3.5) reproduced here:

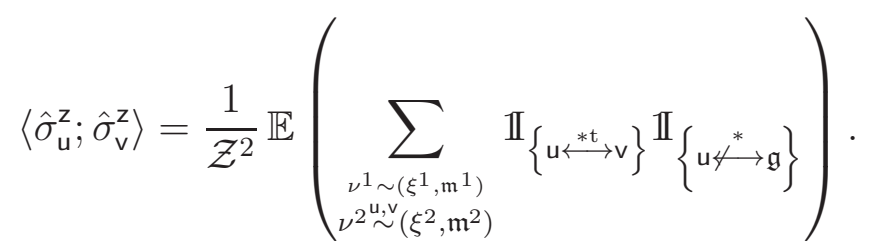

There is a simple reason to include a redundant constraint $\{\mathrm{u} \stackrel{* t}{\longleftrightarrow} \mathrm{v}\}$ : Given a realization of $\xi^{1}$ and $\xi^{2}$, the function

$$
\left(\mathfrak{m}^{1}, \mathfrak{m}^{2}\right) \mapsto \sum_{\substack{\nu^{1} \sim\left(\xi^{1}, \mathfrak{m}^{1}\right) \\ \nu^{2} \stackrel{\mathrm{u}, \mathrm{v}}{\sim}\left(\xi^{2}, \mathfrak{m}^{2}\right)}} \mathbb{I}_{\{\mathrm{u} \stackrel{* t}{\longleftrightarrow} \mathrm{v}\}}
$$


is monotone non-increasing. Consequently, for any $F\left(\mathfrak{m}^{1}, \mathfrak{m}^{2}\right)$ non-decreasing, the FKG property of the pair of Poisson processes $\mathfrak{m}^{1}$ and $\mathfrak{m}^{2}$ imply:

$$
\begin{aligned}
& \mathbb{E}\left(F\left(\mathfrak{m}^{1}, \mathfrak{m}^{2}\right) \sum_{\substack{\nu \sim\left(\xi^{1}, \mathfrak{m}^{1}\right) \\
\nu^{2} \sim, v\left(\xi^{2}, \mathfrak{m}^{2}\right)}} \mathbb{I}_{\{\mathrm{u} \stackrel{* t}{\longleftrightarrow} \mathrm{v}\}}\right) \\
& \leq \mathbb{E}\left(F\left(\mathfrak{m}^{1}, \mathfrak{m}^{2}\right)\right) \mathbb{E}\left(\sum_{\nu^{1} \sim\left(\xi^{1}, \mathfrak{m}^{1}\right)} \sum_{\nu^{2} \stackrel{\mathrm{u}, v}{\sim}\left(\xi^{2}, \mathfrak{m}^{2}\right)} \mathbb{1}_{\{\mathrm{u} \stackrel{* \mathrm{t}}{\longleftrightarrow} \mathrm{v}\}}\right) .
\end{aligned}
$$

For every $\delta>0$ fixed (for convenience we'll assume that $\delta$ divides $\beta$ ) let $\mathbb{Z}_{\delta}^{\beta} \triangleq$ $\delta \mathbb{Z} /((\beta / \delta) \mathbb{Z})$ be the rescaled one-dimensional lattice torus which is just an equal $\delta$-spacing embedding of $\beta / \delta$ sites into $\mathbb{S}_{\beta}$.

We construct non-decreasing functions $F_{\delta}\left(\mathfrak{m}^{1}, \mathfrak{m}^{2}\right)=F_{\delta}^{\mathrm{u}, \mathrm{v}}\left(\mathfrak{m}^{1}, \mathfrak{m}^{2}\right)$ as follows: First of all let us map $\mathfrak{S}$ onto $\mathbb{Z}_{\delta}^{\beta} \times \mathbb{Z}^{d}$ : A point $\mathrm{p}=(\delta k, j) \in \mathbb{Z}_{\delta}^{\beta} \times \mathbb{Z}^{d}$ corresponds to the interval $[(k-1) \delta, k \delta)$ of $\mathbb{S}_{\beta}^{j}$. Two points $\mathrm{p}=(\delta k, j)$ and $\mathrm{q}=(\delta l, m)$ are said to be connected if either $j=m$ and $|k-l| \leq 1 \bmod (\beta / \delta)$ or $k=l$ and $(j, m) \in \mathcal{E}$.

Consider the following Bernoulli site percolation process $X_{\delta}$ on $\mathbb{Z}_{\delta}^{\beta} \times \mathbb{Z}^{d}$, which is generated by the combined process of marks $\mathfrak{n}$

$$
X_{\delta}(\mathbf{p})= \begin{cases}0, & \text { if } \mathfrak{n}(j \times[(k-1) \delta, k \delta))>0 \\ \delta, & \text { otherwise }\end{cases}
$$

Clearly, $\mathbb{P}\left(X_{\delta}=\delta\right)$ tends to one as $\delta$ tends to zero. For $\mathrm{p}, \mathrm{q} \in \mathbb{Z}_{\delta}^{\beta} \times \mathbb{Z}^{d}$ we can define the minimal passage time

$$
T_{\delta}(\mathrm{p}, \mathrm{q})=\min _{\gamma_{\delta}: \mathrm{p} \mapsto \mathrm{q}} \sum_{\mathrm{r} \in \gamma} X_{\delta}(\mathrm{r}) .
$$

Then, there exist $c_{1}, c_{2}>0$ such that

$$
\mathbb{P}\left(T_{\delta}(\mathrm{p}, \mathrm{q})<\frac{\delta}{2} \mathrm{~d}_{\delta}(\mathrm{p}, \mathrm{q})\right) \leq c_{2} \mathrm{e}^{-c_{1} \mathrm{~d}_{\delta}(\mathrm{p}, \mathrm{q})},
$$

uniformly in $0 \leq \delta \leq \delta_{0}$ small enough and in $\mathrm{p}, \mathrm{q} \in \mathbb{Z}_{\delta}^{\beta} \times \mathbb{Z}^{d}$. Moreover, our choice of $\delta_{0}$ may be made independent of $\beta$. Here, $\mathrm{d}_{\delta}(\mathrm{p}, \mathrm{q})$ is the minimal possible number of points in connected paths $\gamma_{\delta}: \mathrm{p} \mapsto \mathrm{q}$.

Note that if $\mathrm{p}_{\mathrm{u}}$ and $\mathrm{p}_{\mathrm{v}}$ label $\delta$-intervals containing $\mathrm{u}$ and $\mathrm{v}$, then

$$
\mathrm{d}_{\delta}\left(\mathrm{p}_{\mathrm{u}}, \mathrm{p}_{\mathrm{v}}\right) \geq c_{3} \mathrm{~d}(\mathrm{u}, \mathrm{v})
$$

uniformly in $\delta$ small and, say, $\mathrm{d}(\mathrm{u}, \mathrm{v}) \geq 1$. Suppose that for such $\delta, \mathrm{p}_{\mathrm{u}}$ and $\mathrm{p}_{\mathrm{v}}$, we also assume $\delta>0$ is chosen to satisfy (5.4). If we define

$$
\mathcal{D}_{\delta}^{c}=\left(\mathcal{D}_{\delta}^{\mathrm{u}, \mathrm{v}}\right)^{c}=\left\{T_{\delta}\left(\mathrm{p}_{\mathrm{u}}, \mathrm{p}_{\mathrm{v}}\right)<\frac{\delta}{2} \mathrm{~d}_{\delta}\left(\mathrm{p}_{\mathrm{u}}, \mathrm{p}_{\mathrm{v}}\right)\right\}
$$


then since $F_{\delta} \triangleq \mathbb{I}_{\mathcal{D}_{\delta}^{c}}$ is non-decreasing, the FKG inequality (15.3) along with (5.4) imply that for all $\delta$ small there exist $c_{1}=c_{1}(\delta), c_{2}>0$, such that

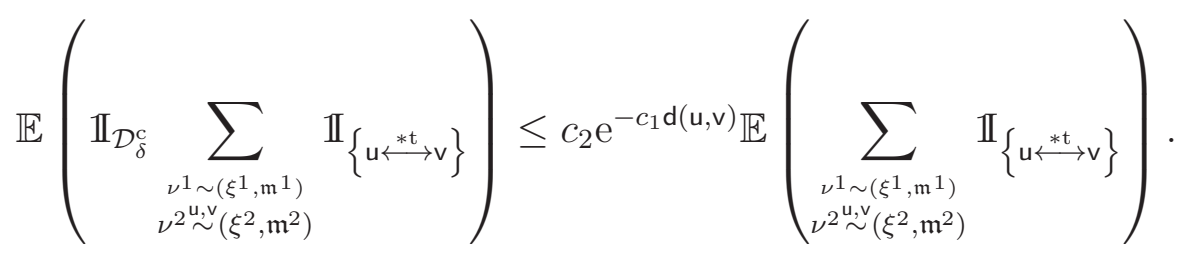

In view of (5.5) it suffices to check that, perhaps by adjusting further $c_{1}, c_{2}>0$,

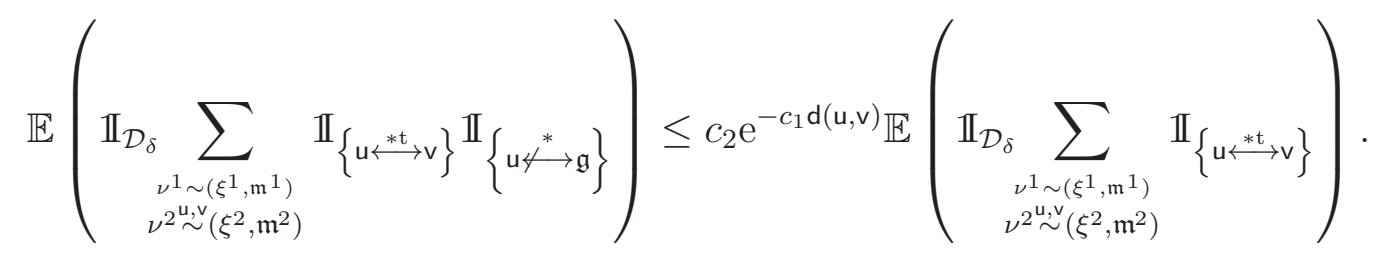

Consider now the set $\mathfrak{A}^{* \mathfrak{t}}(\mathrm{u}, \mathrm{v})$ of all the points $z \in \mathfrak{S}$ which are $* \mathfrak{t}$-connected to both $\mathrm{u}$ and $\mathrm{v}$ (see above Figure 2 for the definition). The set $\mathfrak{A}^{* \mathfrak{t}}(\mathrm{u}, \mathrm{v})$ is non-empty on the event $\{\mathrm{u} \stackrel{* t}{\longleftrightarrow} \mathrm{v}\}$, and it is represented as a union of intervals $\mathfrak{A}^{* \mathfrak{t}}(\mathrm{u}, \mathrm{v})=\cup_{l} \mathfrak{R}_{l}$. Each interval $\mathfrak{R}_{l} \subset \mathbb{S}_{\beta}^{l}$ is either empty (this case is included for notational convenience), or it is a full circle, or $\mathfrak{R}_{l}=\left(\mathrm{z}_{l}, \mathrm{w}_{l}\right) \subset \mathbb{S}_{\beta}^{i_{l}}$ with combined $\mathfrak{n}$ marks placed at both end-points (it could happen that $\mathrm{z}_{l}=\mathrm{w}_{l}$, of course). Note that these endpoints must also have $\nu^{1}, \nu^{2}=r$.

Let us say that $\mathrm{p}=(\delta k, l) \in \mathfrak{G}_{\delta}\left(\mathfrak{R}_{l}\right)$ if

$$
\mathfrak{R}_{l} \neq \emptyset, \mathrm{p} \in \mathfrak{R}_{l} \quad \text { and } \quad X_{\delta}(\mathrm{p})=\delta .
$$

Note that $\mathrm{p} \in \mathfrak{G}_{\delta}\left(\mathfrak{R}_{l}\right)$ implies in particular that $[(k-1) \delta, k \delta) \times l \subseteq \mathfrak{R}_{l}$.

The crucial property is that on the event $\mathcal{D}_{\delta}^{u, v}$ the following happens: The number of all $\delta$-intervals associated with points $\mathrm{p} \in \cup_{l} \mathfrak{G}_{\delta}\left(\mathfrak{R}_{l}\right)$ is bounded below as

$$
\sum_{l} \sum_{\mathrm{p} \in \mathfrak{G}_{\delta}\left(\Re_{l}\right)} \mathrm{I} \geq \frac{1}{\delta} T_{\delta}\left(\mathrm{p}_{\mathrm{u}}, \mathrm{p}_{\mathrm{v}}\right)>c_{3} \frac{1}{2} \mathrm{~d}(\mathrm{u}, \mathrm{v}) .
$$

Let us condition on realizations of $\mathfrak{A}^{* \mathfrak{t}}(\mathrm{u}, \mathrm{v})$ which are compatible with $\{\mathrm{u} \stackrel{* t}{\longleftrightarrow} \mathrm{v}\}$ and $\mathcal{D}_{\delta}^{u, v}$. As before, such a conditioning rules out simultaneous flips between points in $\mathfrak{A}^{* \mathfrak{t}}(\mathrm{u}, \mathrm{v})$ and $\mathfrak{S} \backslash \mathfrak{A}^{* \mathfrak{t}}(\mathrm{u}, \mathrm{v})$. Therefore, the corresponding conditional integration and summation over compatible flips, marks and labels inside and outside $\mathfrak{A}^{* \mathfrak{t}}(\mathrm{u}, \mathrm{v})$ decouples over the two regions.

In other words, to establish (5.6) it is enough to prove the following statement: Let $\mathfrak{A}=\cup \mathfrak{R}_{l}$ be a collection of disjoint intervals, such that $\mathrm{u}$ and $\mathrm{v}$ are interiour points of $\mathfrak{A}$. Further, suppose that $\mathfrak{A}$ contains at least $c_{3} \frac{1}{2} \mathrm{~d}(\mathrm{u}, \mathrm{v})$ disjoint sub-intervals each with length at least $\delta$ and let us say that $\mathcal{D}_{\delta}^{\mathrm{u}, \mathrm{v}}(\mathfrak{A})$ occurs for the realization of the combined process of marks $\mathfrak{n}$ whenever (5.7) holds. 
Let $\rho^{\mathfrak{A}}$ denote the reduced time-inhomogeneous rates of arrivals of flips (associated to edges on the torus) as in (3.17),

$$
\rho_{\mathrm{e}}^{\mathfrak{A}}(t)= \begin{cases}\rho, & \text { if the corresponding flip is either between two points in } \mathfrak{A} \\ & \text { or between two points in } \mathfrak{S} \backslash \mathfrak{A} \\ 0, & \text { otherwise. }\end{cases}
$$

Then,

$$
\begin{aligned}
& \mathbb{E}_{\rho^{\mathfrak{A}}} \sum_{\substack{\check{\nu}^{1} \sim\left(\xi^{1}, \mathfrak{m}^{1}\right) \\
\check{\nu}^{2} \stackrel{\mathrm{u} v}{\sim}\left(\xi^{2}, \mathfrak{m}^{2}\right)}} \mathbb{I}_{\left\{\mathrm{C}^{* \mathfrak{t}}(\mathrm{u}, \mathrm{v})=\mathfrak{A}\right\}} \mathbb{I}_{\left\{\mathrm{u} \psi^{*} \rightarrow \mathfrak{g}\right\}} \mathbb{I}_{\left\{\mathcal{D}_{\delta}^{\mathrm{u}, \mathrm{v}}(\mathfrak{A})\right\}} \\
& \leq c_{2} \mathrm{e}^{-c_{1} \mathrm{~d}(\mathrm{u}, \mathrm{v})} \mathbb{E}_{\rho^{\mathfrak{A}}} \sum_{\substack{\check{\nu}^{1} \sim\left(\xi^{1}, \mathfrak{m}^{1}\right) \\
\check{\nu}^{2} \stackrel{\mathrm{uv}}{\sim}\left(\xi^{2}, \mathfrak{m}^{2}\right)}} \mathbb{I}_{\left\{\mathrm{C}^{* \mathrm{t}}(\mathrm{u}, \mathrm{v})=\mathfrak{A}\right\}} \mathbb{I}_{\left\{\mathcal{D}_{\delta}^{\mathrm{u}, \mathrm{v}}(\mathfrak{A})\right\}},
\end{aligned}
$$

where $\check{\nu}^{1}, \check{\nu}^{2}$ are restrictions of the labels to $\mathfrak{A}=\cup \mathfrak{R}_{l}$ which are compatible with the marks, and in particular with $r$-boundary conditions, at the end-points of $\mathfrak{R}_{l}$-s. Above $\mathrm{C}^{* \mathrm{t}}(\mathrm{u}, \mathrm{v})$ is the set of points which are $* \mathrm{t}$-connected to $\mathrm{u}$ and $\mathrm{v}$.

The inequality (5.9) is established by the following embedding procedure: Let $\left\{\left(\check{\nu}^{1}, \xi^{1}, \mathfrak{m}^{1}\right),\left(\check{\nu}^{2}, \xi^{2}, \mathfrak{m}^{2}\right)\right\}$ be a pair of configurations which contribute to the left hand side of (5.9). All such configurations have no arrivals of $\mathfrak{g}$-induced flips on $\mathfrak{A}$. At this stage it is convenient to introduce the following separate notation for processes of flips: let $\check{\xi}_{\mathrm{e}}^{k} ; k=1,2$, to denote arrivals for $\mathrm{e}=(i, j) \in \mathcal{E}^{0}$ and $\xi_{\mathrm{e}}^{\mathfrak{g}, k} ; k=1,2$, to denote arrivals for $\mathrm{e}=(i, \mathfrak{g}) \mathcal{E}^{\mathfrak{g}}$. A similar notation $\check{\eta}=\check{\xi}^{1} \cup \check{\xi}^{2}$ and $\eta^{\mathfrak{g}}=\xi^{\mathfrak{g}, 1} \cup \xi^{\mathfrak{g}, 2}$ is introduced for combined processes of flips. Then, on the event $\eta^{\mathfrak{g}}(\mathfrak{A})=\emptyset$, the compatibility conditions on the left hand side of (5.9) read as $\check{\nu}^{1} \sim\left(\check{\xi}^{1}, \mathfrak{m}^{1}\right)$ and, accordingly, $\check{\nu}^{2} \stackrel{\text { u,v }}{\sim}\left(\check{\xi}^{2}, \mathfrak{m}^{2}\right)$, and the expression on the left hand side of ([5.9) equals to

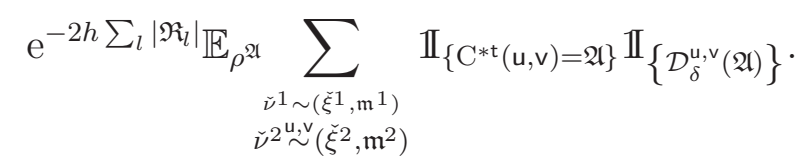

Fix now a realization of $\left(\check{\xi}^{1}, \mathfrak{m}^{1}\right),\left(\check{\xi}^{2}, \mathfrak{m}^{2}\right)$ and compatible labels $\check{\nu}^{1} \sim\left(\check{\xi}^{1}, \mathfrak{m}^{1}\right)$ and $\check{\nu}^{2} \stackrel{u, v}{\sim}\left(\check{\xi}^{2}, \mathfrak{m}^{2}\right)$. Consider the following event

$$
\mathbf{E}(\mathfrak{A})=\cap_{l} \cap_{\mathbf{p} \in \mathfrak{G}_{\delta}\left(\mathfrak{R}_{l}\right)}\left\{\xi^{\mathfrak{g}, i}\left(\mathfrak{I}_{\mathbf{p}}\right) \text { is even for } i=1,2\right\} \cap\left\{\eta^{\mathfrak{g}}\left(\mathfrak{A} \backslash \mathfrak{A}_{\delta}\right)=0\right\},
$$

where, for $\mathrm{p}=(k \delta, l) \in \delta \mathbb{Z}_{\delta}^{\beta} \times \mathbb{Z}^{d}$, we set

$$
\mathfrak{I}_{\mathrm{p}}=[(k-1) \delta, k \delta) \times l \quad \text { and } \quad \mathfrak{A}_{\delta}=\cup_{l} \cup_{\mathrm{p} \in \mathfrak{G}_{\delta}\left(\mathfrak{R}_{l}\right)} \mathfrak{I}_{\mathrm{p}} .
$$

Evidently,

$$
\mathbb{P}(\mathbf{E}(\mathfrak{A}))=\mathrm{e}^{-2 h \sum_{l}\left|\mathfrak{R}_{l}\right|} \prod_{l} \prod_{\mathbf{p} \in \mathfrak{G}_{\delta}\left(\mathfrak{R}_{l}\right)}(\cosh (\delta h))^{2} \geq \mathrm{e}^{-2 h \sum_{l}\left|\mathfrak{R}_{l}\right|}(\cosh (\delta h))^{c_{3} \mathrm{~d}(\mathrm{u}, \mathrm{v})}
$$

where the second inequality follows from (5.7). Each $\mathbf{E}(\mathfrak{A})$-realization of $\left(\xi^{\mathfrak{g}, 1}, \xi^{\mathfrak{g}, 2}\right)$ gives rise to compatible labels $\check{\nu}^{1}\left[\xi^{\mathfrak{g}, 1}\right] \sim\left(\check{\xi}^{1}, \xi^{\mathfrak{g}, 1}, \mathfrak{m}^{1}\right)$ and $\check{\nu}^{2}\left[\xi^{\mathfrak{g}, 2}\right] \stackrel{\mathrm{u}, \mathfrak{v}}{\sim}\left(\check{\xi}^{2}, \xi^{\mathfrak{g}, 2}, \mathfrak{m}^{2}\right)$ which are unambiguously constructed from the original $\check{\nu}^{1} \sim\left(\check{\xi}^{1}, \mathfrak{m}^{1}\right)$ and $\check{\nu}^{2} \stackrel{\text { u,v }}{\sim}$ 
$\left(\check{\xi}^{2}, \mathfrak{m}^{2}\right)$ by the appropriate even number of flips on each of the intervals $\mathfrak{I}_{\mathrm{p}} \subseteq \mathfrak{A}_{\delta}$ (see Figure 7).

(a)

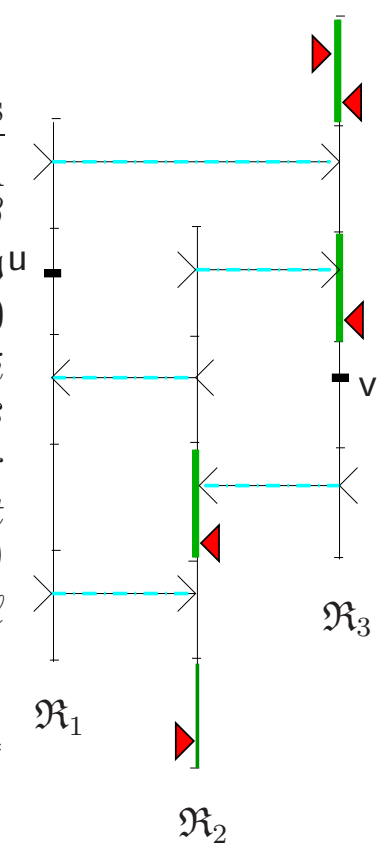

$>\quad$ Ground flips and marks $\left(\check{\xi}^{1}, \mathfrak{m}^{1}\right) \quad \mid$ Intervals with $X_{\delta}=0$

$<\triangleleft$ Ground flips and marks $\left(\check{\xi}^{2}, \mathfrak{m}^{2}\right)$

Figure 7. Compatible labels which are constructed from $\check{\nu}^{1}, \check{\nu}^{2}$ and even number of arrivals of $\xi^{\mathfrak{g}, 1}$ and $\xi^{\mathfrak{g}, 2}$ on intervals from $\mathfrak{G}_{\delta}$ :

(a) Original configurations $\left(\check{\nu}^{1}, \check{\xi}^{1}, \mathfrak{m}^{1}\right)$ and $\left(\check{\nu}^{2}, \check{\xi}^{2}, \mathfrak{m}^{2}\right)$.

(b) Example of admissible (in the sense of event $\mathbf{E}$ ) even number of arrivals of $\left(\xi^{\mathfrak{g}, 1}, \xi^{\mathfrak{g}, 2}\right)$ : the circled numbers indicate total number of arrivals on the corresponding intervals.

As a result, the expectation on the right-hand side of (5.9) is bounded below by

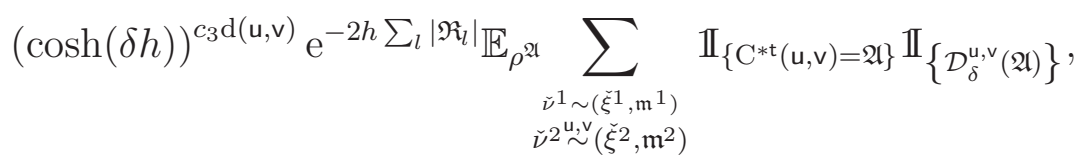

and (5.9) follows.

Proof of Theorem A for xz-correlations. Recall the expression (3.12),

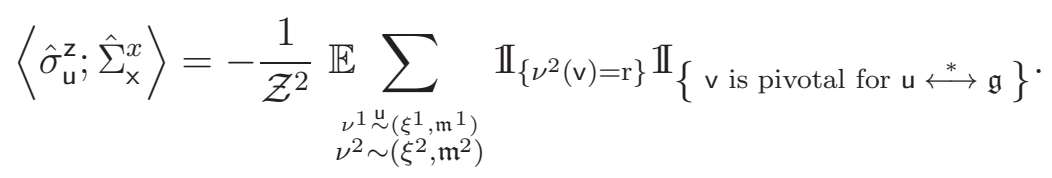


Observe that if $\mathbf{v}$ is pivotal for $\mathbf{u}^{*} \longleftrightarrow \mathfrak{g}$ then $\mathfrak{A}^{*}(\mathrm{u}, \mathrm{v})$ (recall that the latter notation stand for the set of points which are $*$-connected to $u$ by paths avoiding $v$ ) does not contain $\mathfrak{g}$, which means that there are no arrivals of $\eta^{\mathfrak{g}}$ on $\mathfrak{A}^{*}(\mathrm{u}, \mathrm{v})$. At this point we may proceed exactly as in the proof of Theorem A for z-correlations.

Proof of Theorem A for $\mathrm{x}$-correlations. Recall the expression (3.9)

$$
\left\langle\hat{\Sigma}_{\mathrm{u}}^{x} ; \hat{\Sigma}_{\mathrm{v}}^{x}\right\rangle=\frac{1}{\mathcal{Z}^{2}} \mathbb{E} \sum_{\substack{\nu^{1} \sim\left(\xi^{1}, \mathfrak{m}^{1}\right) \\ \nu^{2} \sim\left(\xi^{2}, \mathfrak{m}^{2}\right)}} \mathbb{I}_{\left\{\left(\nu^{1}, \nu^{2}\right) \in[(\mathrm{r}, 1),(\mathrm{r}, 1)]\right\}} \mathbb{I}_{\{\mathrm{v} \text { is loop pivotal for } \mathrm{u}\}} .
$$

Observe that under the constraints on the right-hand side, if $v$ is loop-pivotal for $\mathrm{u}$ then the set $\mathfrak{A}^{*}(\mathrm{u}, \mathrm{v}) \backslash\{\mathrm{u}, \mathrm{v}\}$ contains at least two disjoint components. Hence at least one of these components should be disjoint from $\mathfrak{g}$. Again, at this point we may proceed exactly as in the proof of Theorem A for z-correlations.

Implications for the ground state $\beta=\infty$. As was proved above, exponential decay of truncated two-point functions is uniform in $\beta<\infty$. Consequently, for every $N<\infty$, the limit

$$
M_{\infty, N}(h, \rho, \lambda) \triangleq \lim _{\beta \rightarrow \infty} M_{\beta, N}(h, \rho, \lambda)
$$

also satisfies (1.3) and (1.4). On the other hand, by an obvious time scaling, $M_{\infty, N}(\alpha h, \alpha \rho, \alpha \lambda)=M_{\infty, N}(h, \rho, \lambda)$ for every $\alpha>0$. Hence,

$$
\rho \frac{\partial M_{\infty, N}}{\partial \rho}=-\lambda \frac{\partial M_{\infty, N}}{\partial \lambda}-h \frac{\partial M_{\infty, N}}{\partial h} \leq-\lambda \frac{\partial M_{\infty, N}}{\partial \lambda}
$$

Therefore, (1.3) implies that

$$
M_{\infty, N} \leq h \frac{\partial M_{\infty, N}}{\partial h}+M_{\infty, N}^{3}-3 M_{\infty, N}^{2} \lambda \frac{\partial M_{\infty, N}}{\partial \lambda} .
$$

Together with the first of (1.4) (for $M_{\infty, N}$ ) the inequality (5.14) sets up the stage for an analysis of sharpness of of the $\hat{\sigma}^{z}$ phase transition literally along the lines of $[2,3]$.

Acknowledgement. Our proof of exponential decay is based on an argument which was developed in the classical setting together with Roberto Fernandez and Yvan Velenik (see [15]). We are grateful to Anna Levit for useful remarks and a very careful reading of the first draft of this paper.

\section{REFERENCES}

[1] M. Aizenman. Geometric analysis of $\phi^{4}$ fields and Ising models. Commun. Math. Phys., 86(1), 1982.

[2] M. Aizenman and D. J. Barsky, Sharpness of the phase transition in percolation models. Comm. Math. Phys., 108(3), 489-529, 1987.

[3] M. Aizenman, D. J. Barsky, and R. Fernández. The phase transition in a general class of Ising-type models is sharp. J. Statist. Phys., 47(3-4):343-374, 1987.

[4] M. Aizenman and R. Fernández. On the critical behavior of the magnetization in highdimensional Ising models. J. Statist. Phys., 44(3-4):393-454, 1986.

[5] M. Aizenman, A. Klein, and C. Newman. Percolation methods for disordered quantum Ising models. In R. Kotecky, editor, Phase Transitions: Mathematics, Physics, Biology,.., pages 1-26. World Scientific, Singapore, 1993. 
[6] M. Aizenman and B. Nachtergaele. Geometric aspects of quantum spin states. Commun. Math. Phys., 164:17-63, 1994.

[7] M. Biskup, L. Chayes, and N. Crawford. Mean-field driven first-order phase transitions in systems with long-range interactions. J. Statist. Phys., 119(6):1139-1193, 2006.

[8] J. E. Björnberg and G. Grimmett, The phase transition of the quantum Ising model is sharp, Preprint, http://xxx. lanl.gov/pdf/0901.0328, 2009.

[9] M. Campanino, A. Klein, and J.F Perez. Localization in the ground state of the Ising model with a random transverse field. Commun. Math. Phys., 135:499-515, 1991.

[10] L. Chayes, N. Crawford, D. Ioffe, and A. Levit. The phase diagram of the quantum CurieWeiss model. Preprint, to appear in J. Statist. Phys., http://arxiv.org/pdf/0804.1605, 2008.

[11] N. Crawford and D. Ioffe. In preparation, 2009.

[12] J. Ginibre. Existence of phase transitions for quantum lattice systems. Commun. Math, Phys., 14:205-234, 1969.

[13] R. Griffiths. Correlations in Ising Ferromagnets. II J. Math. Phys., 8:484, March 1967.

[14] R. Griffiths, C. Hurst, and S. Sherman. Concavity of magnetization of an Ising ferromagnet in a positive external field. J. Math. Phys., 11:790, March 1970.

[15] D. Ioffe. Stochastic geometry of classical and quantum Ising models. In to appear in Lecture Notes in Mathematics. Springer.

[16] D. Ioffe and A. Levit. Long range order and giant components of quantum random graphs. Markov. Proc. Rel. Fields, 13(3):469-492, 2007.

[17] S. Shlosman. Signs of Ursell's functions. Commun. Math. Phys., 102(4):679-686, 1985.

Department of Statistics, UC Berkeley; Berkeley, CA

Faculty of Industrial Engineering, Technion, Haifa 3200, Israel

E-mail address: ieioffe@ie.technion.ac.il 